Research Article

\title{
Research on the Risk Assessment of New Energy Automobile Industry Based on Entropy Weight-Cloud Model in China's Jiangsu Province
}

\author{
Yanqiu Cao $\mathbb{D}^{1,2,3}$ Yijie Bian, ${ }^{1,3}$ Rong Wang, ${ }^{4}$ and Lingjun Wang ${ }^{5}$ \\ ${ }^{1}$ Business School, Hohai University, Nanjing 211100, China \\ ${ }^{2}$ Jincheng College, Nanjing University of Aeronautics and Astronautics, Nanjing 211156, China \\ ${ }^{3}$ Jiangsu Provincial Collaborative Innovation Center of World Water Valley and Water Ecological Civilization, Nanjing, \\ Jiangsu 211100, China \\ ${ }^{4}$ Business School, Nanjing Xiaozhuang University, Nanjing, Jiangsu 211171, China \\ ${ }^{5}$ Nanjing Institute of Technology, Nanjing, Jiangsu 211167, China
}

Correspondence should be addressed to Yanqiu Cao; thinking@nuaa.edu.cn

Received 18 April 2020; Revised 15 September 2020; Accepted 28 May 2021; Published 8 June 2021

Academic Editor: Reza Hemmati

Copyright (c) 2021 Yanqiu Cao et al. This is an open access article distributed under the Creative Commons Attribution License, which permits unrestricted use, distribution, and reproduction in any medium, provided the original work is properly cited.

The development of the new energy vehicle industry is necessary for its advantages of saving energy and reducing greenhouse-gas emissions. However, the industry is currently facing risks with regard to, for example, technology, market, and the policy. Most existing studies of industry risk focused on analyzing and evaluating risk factors and summarizing and interpreting risk phenomena. In this study, systematic classification and quantitative analysis for the risk of the new energy vehicle industry were investigated, in which the entropy weight method and cloud model were combined to evaluate and quantify the industry risk. The formation mechanism of the industry risk from endogenous and exogenous perspectives was analyzed to screen out riskevaluation factors. Combining the expert-investigation and fuzzy-statistics methods, a risk-evaluation index system with six primary indicators and twenty-four secondary indicators was constructed. On the basis of the entropy weight-cloud model, the risk of the new energy automobile industry of Jiangsu province in July 2019 was evaluated. Results indicated that the impact of exogenous risk on the industry was greater than that of endogenous risk, and industry risk was higher than medium risk, which was close to a higher medium level. A series of suggestions are given for preventing industry risk, such as improving the industry's own ability to resist risk and building the industry's soft environment.

\section{Introduction}

With the rapid development of the economy and the acceleration of the urbanization process, automobiles have had rapidly expanded outputs, sales, and ownership in China, resulting in more resource exhaustion and environmental pollution. To reduce these disadvantages, developing a new energy automobile industry with the characteristics of energy conservation, environmental protection, and low carbon consumption is necessary, since it can help reduce greenhouse-gas emissions and improve the global ecological environment [1]. The new energy automobile industry in Jiangsu province is one of the key industries in the strategic emerging-industry development plan of the 13th five-year plan of Jiangsu. In 2017, the sales revenue of new energy vehicles and key parts and components reached 45.59 billion yuan in Jiangsu, ranking it first in China. In 2018, the production and sales of new energy vehicles increased rapidly. Total sales of new energy vehicles in Jiangsu were 164,000 , a year-on-year increase of $158.2 \%$, accounting for $13.1 \%$ of China's total sales. The installed capacity of power batteries was $14.8 \mathrm{GWh}$, a year-on-year increase of $66.3 \%$, accounting for $24.5 \%$ of the whole country. On the basis of the above-mentioned data, the development of new energy vehicles in Jiangsu is necessary. Moreover, the new energy automobile industry has characteristics of a long industrial 
chain, high degree of correlation, and strong driving force, which can promote the development of upstream and downstream industries, indicating that it will become the growth point of future economy in Jiangsu [2]. However, this industry is currently facing many risk types, with regard to technology, the market, and policy. The lack of an appropriate industry risk-assessment method and failing to control risk on the basis of assessment results affects the development of the industry in Jiangsu and even the entire Chinese economy. Therefore, it is necessary to evaluate the risk of the new energy vehicle industry and prevent it on the basis of assessment results, so as to promote the green and sustainable development of the industry.

This study aims to (1) construct an industry-risk-evaluation index system from endogenous and exogenous perspectives and propose a risk-assessment method that combines the entropy weight method and cloud model; (2) evaluate the new energy automobile industry risk of Jiangsu on the basis of the entropy weight-cloud model; and (3) propose a reference for risk prevention of the new energy automobile industry in Jiangsu.

The main contributions of this article are shown in two aspects. First, it analyzes the industry risk formation mechanism through both an endogenous and an exogenous perspective and then combines the expert-analysis and fuzzy-statistical methods to screen out endogenous-risk and exogenous-risk evaluation factors. On the basis of the above in-depth analysis and digging, the construction of the riskassessment index system is more reasonable. Furthermore, this study offers an effective approach for evaluating the risk of the new energy vehicle industry. The cloud model is introduced into industry risk assessment, which considers risk randomness and ambiguity, thus achieving the transformation of qualitative concepts to quantitative indicators and guaranteeing the objectivity and accuracy of the assessment results.

\section{Literature Review}

2.1. Research on the Risk of the New Energy Vehicle Industry. Little investigation has reported the risk of the new energy automobile industry abroad, which was primarily focused on the perspective of strategic emerging-industry risk. Domestic research on the risks of the new energy vehicle industry has arisen in recent years. Many scholars have identified and analyzed risk factors. From the point of view of the patent, Li et al. [3] carried out an investigation about the positioning and transferring of integrated-equipmentmanufacturing (IDM) technology focus in order to predict the technology-replacement risk of a strategic emerging industry generated from the nonmainstream or competition. In the principle of the Delphi method, Christoph [4] conducted an experiment on the identification and evaluation of future supply-chain safety problems under the situation of the multiple stakeholders. Liu and Cheng [5] identified exploratory-factor and principal-component analyses by studying questionnaires and explained that technical factor was the key demand risk factor for Chinese new energy vehicles. Wang et al. [6] evaluated uncertainly influencing factors of the global supply risk of key raw materials for new energy vehicles by the Monte Carlo method. Zhang and Su [7] proposed key elements of evaluating the new energy vehicle venture-investment project, including the industrial environment, economic environment, product performance, and key technology competitiveness. Studies on categorized risks have been reported. Chen et al. [8] classified the innovation-cluster Internet risk as exogenous and endogenous risks. Wu and Liu [9] put forward the systemfragile, blind-expansion, and match-reliance risks of the new energy vehicle innovation-ecology system existing during the period of three evolution stages. Su et al. [10] divided the explosion risk of new energy vehicles, including vehiclecontrol-, auxiliary-, and power-system safety. The literature on risk assessment is currently scarce. Zhang and Sun [7] proposed a G1-ENTROPY-TOPSIS evaluation method to assess new energy vehicle venture-investment projects. On the basis of the aforementioned literature, most studies mainly focused on the identification and analysis of risk factors, as well as the empirical induction and explanation of the risk phenomena. Deeply systematic classification and quantitative analysis of various risks in the new energy automobile industry are few, which is not conducive to the development of an industry-risk management system. There is a lack of research on risk-assessment methods for the new energy vehicle industry, which is proposed after deeper digging into industry risk on the basis of the current Chinese situation.

2.2. Risk Evaluation Methods. Common risk-evaluation methods include the probabilistic-risk-assessment [11], scoring [12], risk-matrix [13], Petri Nets (PN) [14], TOPSIS [15], fuzzy-comprehensive-evaluation (FCEM) methods $[16,17]$, rough-set theory [18], and hedging instruments [19]. These methods were specifically induced in application, but they have a variety of disadvantages. The probabilisticrisk-assessment method is complicated in actual applications, and not comprehensive to risk randomness. Scoring methods do not consider the cognitive biases of different users and rarely consider risk correlations, resulting in the invalid evaluation results. The risk-matrix method is highly subjective in judging standards of risk importance, the possibility of risk occurrence, and the severity of consequences, which cannot obtain the importance level of the overall risk through mathematical operations on individual risk-importance levels. The main limitation of the PN riskassessment method is that deep knowledge of system analysis is required, but it is not simple to build the net. TOPSIS orders risk-assessment programs according to the closeness of a limited number of evaluation objects to the idealized target, while being unable to rank risks. The fuzzy evaluation matrix obtained by the FCEM is often not unique and may result in the super-fuzzy phenomenon occurring. Rough-set theory eliminates redundant data and makes simplified data information content incomplete, which reduces fault tolerance and data promotion to a certain extent. Hedging is only a risk-management technique. All the above methods have the disadvantage that it is difficult to 
overcome the problem of information distortion during the actual evaluation process, since risk-assessment information is transformed into specific numbers, and uncertain decisions are transformed into precise categories. This violates the ambiguity and randomness characteristics of risk. Recently, the cloud model was successfully developed. Compared to the above-mentioned methods, this model can qualitatively describe concepts on the basis of three digital features $\left(E_{x}, E_{n}, H_{e}\right)$ of the cloud and then transform a quantitative description [20], which effectively combines fuzziness in fuzzy-set theory with randomness in probability, making qualitative concept meet quantitative indicators [21]. Unlike the above common risk-assessment methods, the cloud model allows for the membership degree to randomly fluctuate within a certain range of central values rather than a fixed number, thereby subscribing the final information as many cloud images instead of a single value. Therefore, the evaluation conclusion is clearer and more intuitive and detailed, which has great guiding significance for industry risk control [22-24]. Moreover, this method can describe the actual situation of both each risk factor and overall industry risk. Hence, it is a more efficient and convenient method for actual applications.

2.3. Applicability of the Cloud Model. The cloud model has been used in various fields, such as data mining [25], knowledge representation [26], evolutionary algorithms [27], intelligent control [28], network security [29], and decision making $[30,31]$. Additionally, it was introduced to different areas of risk assessment, such as equipmentmanufacturing-industry-chain [32], potential transformer failure [33], slope [34], and stay-cable-durability risks [35], and the cost risk of water projects. The cloud model can be applied to industry risk assessment. First, the three eigenvalues are used to reflect the membership relationship between risk factors and levels, thus effectively implementing the conversion from qualitative concepts to quantitative indicators, ensuring the objectivity of the evaluation results. Second, the cloud model blurs and magnifies boundaries of the risk-level comment set, which is drawn as a cloud map rather than a real number, clarifying evaluation results and making them more intuitive and accurate. Third, the cloud drop distribution of various risk factors is compared in the assessment assembly cloud image, which is convenient in evaluating the industry risk. Therefore, in this study, the cloud model was used for the industry risk assessment of new energy vehicles in which characteristic parameters of the cloud model reflected the size of the industry risk.

This paper consists of five sections. Section 3 expounds the basic theory, including the entropy weight method and cloud model, and then proposes the risk-assessment method based on the entropy weight-cloud model. Section 4 is empirical research on evaluating industry risk. First, it analyzes the formation mechanism of industry risk from endogenous and exogenous perspectives, combining the expert-investigation and fuzzy-statistics methods, and then constructs an industry-risk-assessment index system. Second, it evaluates the new energy vehicle industry risk in Jiangsu province of China with the mode of the entropy weight-cloud. Third, it expounds the assessment process of industry risk and discusses the implication of the results. Lastly, some suggestions are given for preventing industry risk. Section 5 summarizes the research method and offers the research conclusion of this paper.

\section{Methods}

3.1. Cloud Model. The cloud model is a mathematical model for the qualitative and quantitative transformation of uncertain knowledge. Quantitative discourse domain $U$ is expressed by exact numeric values, and the qualitative concept on $U$ is marked as $C$. The random realization of $C$ is $x$, with $x \in C$. It is set so that $u(x) \in[0,1]$ is the membership degree of quantitative value $x$ to $C$, and $u(x)$ is a random number with stabilization bias. $x \in C$ has $x \longrightarrow u(x)$, so the distribution of $x$ on $U$ is referred to as the membership cloud, or cloud for short, and is marked as $C(U)$. There, each $(x, u(x))$ is called as a cloud droplet. The numerical characters of the cloud are expressed by three numerical values, i.e., expectation $\left(E_{x}\right)$, entropy $\left(E_{n}\right)$, and hyper entropy $\left(H_{e}\right)$. Expectation $\left(E_{x}\right)$ is not only the central value of cloud droplets distributed in the space of discourse domain, but also the center of all cloud droplets. Entropy $\left(E_{n}\right)$ refers to the measurement of uncertainty in qualitative concept, which reflects the fuzziness that can be received by a language value in number field and the probability of the language value that can be represented by these points. It is determined by the fuzziness and randomness of matters. Hyper entropy $\left(H_{e}\right)$ reflects the dispersion degree of cloud droplets and the random variation of the membership degree. This article refers to other scholars to build a cloud model as shown in Figure 1 [36].

3.1.1. The Forward Cloud Generator. The forward cloud generator is the course from qualitative concept to quantitative expression, which is the concrete realization for the generation of cloud droplets through the numerical characteristics of the cloud. The rule of the generator is shown in Figure 2.

The algorithm of the forward cloud generator is as follows:

(1) Normal random number $E_{n i}$ is generated with $E_{n}$ as the expected value and $H_{e}$ as variance.

(2) Normal random number $x_{i}$ is generated with $E_{x}$ as the expected value and $E_{n}$ as variance.

(3) It is calculated through

$$
y_{i}=\operatorname{CT}\left(x_{i}\right)=e^{\left(-\left(x i-E_{x}\right)^{2} / 2 E_{n i}^{2}\right)} .
$$

(4) The combination of $\left(x_{i}, y_{i}\right)$ forms a cloud droplet. $x_{i}$ refers to the quantified value of qualitative concept, and $y_{i}$ refers to the certainty of $x_{i}$ belonging to this qualitative concept. 


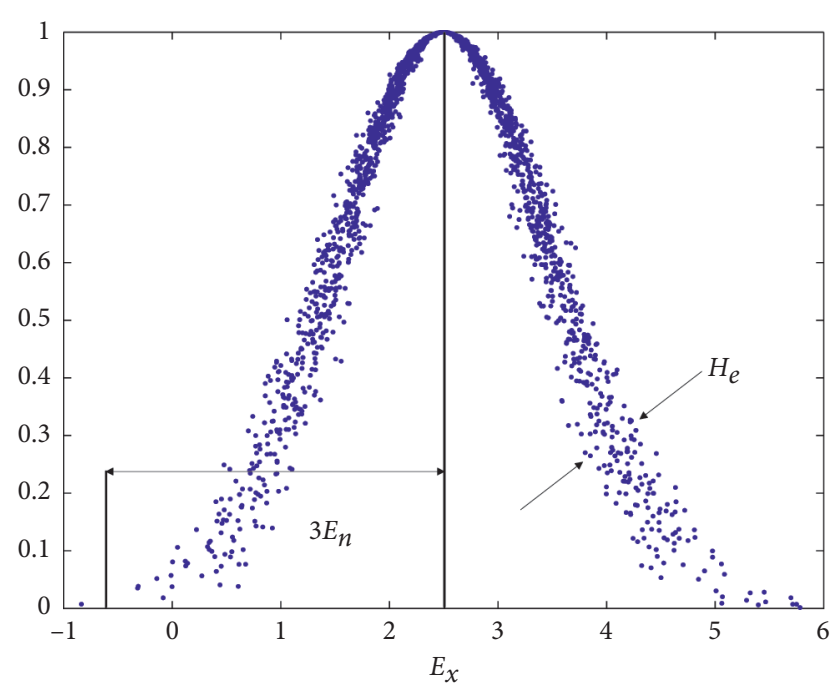

Figure 1: Cloud model and its numerical characters.

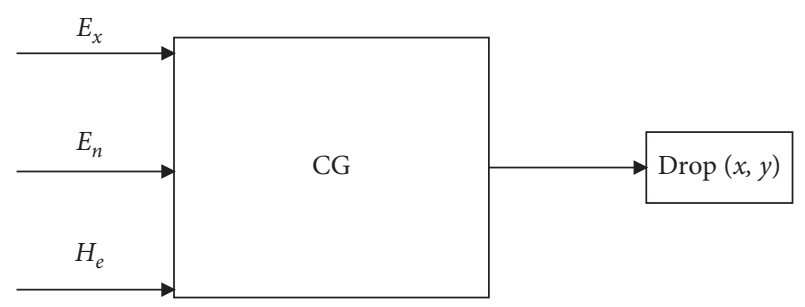

Figure 2: Forward cloud generator.

(5) Steps (1-4) are repeated until the requested number of cloud droplets is generated.

3.1.2. The Backward Cloud Generator. The backward cloud generator is the course from quantitative expression to qualitative concept, which is the concrete realization for the obtainment of numerical characteristics of the cloud through cloud droplets. Its mechanism is shown in Figure 3.

The specific algorithm is shown as follows:

Input: the quantitative value of $n$ cloud droplet(s) and the certainty of concept represented by each cloud droplet, $(x, y)$.

Output: expected value $\left(E_{x}\right)$, entropy $\left(E_{n}\right)$, and hyper entropy $\left(H_{e}\right)$ of the qualitative concept expressed by $\mathrm{n}$ cloud droplet(s).

(1) $E_{x^{\prime}}$ is taken as the estimated value of $E_{x}$ in

$$
E_{x^{\prime}}=\frac{1}{n} \sum_{i=1}^{n} X_{i}
$$

(2) The points of $y>0.999$ are removed, andmis cloud droplet(s) left.

(3) $E_{n^{\prime}}$ is calculated on the basis of

$$
E_{n^{\prime}}=\frac{\left|x-E_{x^{\prime}}\right|}{-\sqrt{2 \ln y}}
$$

(4) The estimated value of $E_{n}$ is calculated by

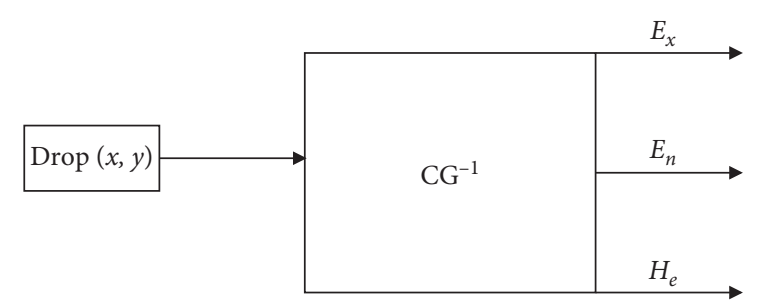

FIgURE 3: Backward cloud generator.

$$
\bar{E}_{n}=\frac{1}{m} \sum_{i=1}^{n} E_{n i}^{\prime}
$$

(5) The estimated value of $H_{e}$ is calculated by

$$
H_{e}=\sqrt{\frac{1}{n-1} \sum_{i=1}^{m}\left(E_{n i}^{\prime}-E_{n}\right)^{2}}
$$

3.1.3. Synthesized Cloud. There may be two or more clouds of the same type generated in the same discourse domain, and these clouds are clustered to generate a synthesized cloud. The essence aims to synthesize several qualitative concepts into a broader concept. The features and numerical characteristics for all syntheses among clouds, i.e., the qualitative variable of $n$-ary, can be expressed by a synthesized cloud. Therefore, a general model is provided for the synthesized cloud among several clouds under normal circumstances. Its numerical characteristics can be expressed by

$$
\left\{\begin{array}{l}
E_{x}=\frac{E_{x 1} \times E_{n 1}+E_{x 2} \times E_{n 2}+\cdots E_{x n} \times E_{n n}}{E_{n 1}+E_{n 2}+\cdots E_{n n}} \\
E_{n}=\frac{E_{n 1}+E_{n 2}+\cdots E_{n n}}{n} \\
H_{e}=\frac{H_{e 1} \times E_{n 1}+H_{e 2} \times E_{n 2}+\cdots H_{e n} \times E_{n n}}{E_{n 1}+E_{n 2}+\cdots E_{n n}},
\end{array}\right.
$$

where $E_{x 1}$ and $E_{n 1}$ represent the expected value and the entropy of the first cloud, respectively. $H_{e 1}$ represents the hyper entropy of the first cloud; $E_{x n}$ represents the expected value of the $\mathrm{n}^{\text {th }}$ cloud; $E_{n n}$ represents the entropy of the $n^{\text {th }}$ cloud; and $H_{e n}$ represents the hyper entropy of the $n^{\text {th }}$ cloud.

3.2. Entropy Weight Method. The entropy weight method is an objective weighting method that can overcome the weight difference caused by subjective factors. Entropy was first introduced by Shannon into information theory, and it was called information entropy [37]. The entropy weight method has been proven to be an effective method for determining the weight with the advantages of precision and objectivity and has also been applied to some practical problems [38-41]. The principle of the entropy weight method is shown as below. The difference between different indices is 
analyzed on the basis of a great deal of information, which is named the degree of variation. First, using information entropy, the entropy weight of each index is calculated on the basis of the degree of index variation. Then, each index is modified by means of entropy weight to obtain the objective and accurate index weight. According to information theory, information is a measurement representing the order degree of the system, and entropy is a measurement representing the disorder degree of system. The smaller information entropy of the indicators can provide more information and play a greater role on comprehensive evaluation, thereby receiving more attention. In this article, the weight of each risk factor in the new energy vehicle industry is determined by the amount of information that affects the risk. In the risk-evaluation index system of the new energy automobile industry constructed in this paper, the weight of each risk factor is determined by the amount of information that can affect this risk. If an index is very controversial among experts, this index has a larger amount of information and a stronger capability in risk identification. Accordingly, this index plays a greater role in comprehensive risk evaluation, so it has a larger weight. The weight of each risk factor in the index can be calculated on the basis of formulas of the entropy weight method, as shown in equations (7)-(11):

$$
y_{i j}=\frac{x_{i j}-\min \left(x_{i}\right)}{\max \left(x_{i}\right)-\min \left(x_{i}\right)} .
$$

Equation (7) is to standardize the experts of each risk factor, and $x_{i j}$ is the score of the risk factor for the $i$-th expert.

$$
P_{i j}=\frac{y_{i j}}{\sum_{i=1}^{m} y_{i j}} .
$$

In equation (8), $P_{i j}$ is the characteristic weight between each risk factor.

$$
e_{j}=-k \sum_{i=1}^{m} p_{i j} \ln p_{i j} .
$$

In equation (9), $e_{j}$ is the entropy value of the risk factor, $0 \leq e_{j} \leq 1 ; \quad k>0, \quad k=1 / \ln m$, and delimit $\lim _{p_{i j}} \longrightarrow 0 p_{i j}$ $\ln p_{i j}=0$

$$
g_{j}=1-e_{j}
$$

In equation (10), $g_{j}$ is the risk difference coefficient.

$$
w_{j}=\frac{g_{j}}{\sum_{j=1}^{n} g_{j}} .
$$

In equation (11), $n$ represents the number of risk factors, and $w_{j}$ is the weight of each risk factors.

3.3. Cloud Entropy Optimization Algorithm. Calculating the characteristic value of the cloud model is a key step in the assessment of industry risk based on cloud model. The traditional extension cloud theory regards hierarchical boundaries as a double-constrained space $\left[c_{\min }, c_{\max }\right]$. It uses the center value of the constraint interval to represent the values of $E_{x}$, as shown in

$$
E_{x}=\frac{c_{\min }+c_{\max }}{2}
$$

$H_{e}$ is generally taken as a constant, which can be adjusted according to the actual experience and uncertainty of the evaluation index. As a measure of the ambiguity of the concept of state level, the size of cloud entropy $E_{n}$ reflects the acceptable numerical range of the concept of state level, which affects the accuracy of the evaluation results. There are mainly two kinds of cloud entropy calculation methods based on scholars' understanding of different angles of classification [42].

3.3.1. Cloud Entropy Computing Method Based on " $3 E_{n}$ " Rule. The " $3 E_{n}$ " rule refers to the fact that the elements of cloud droplets located outside the interval $\left[E_{x}-3 E_{n}, E_{x}+\right.$ $\left.3 E_{n}\right]$ are low-probability events. According to the rule, adjacent hierarchical extension clouds with clear boundaries are obtained, as shown in

$$
E_{n}^{\prime}=\frac{c_{\max }-c_{\min }}{6}
$$

3.3.2. Cloud Entropy Computing Method Based on " $50 \%$ Relevance" Rule. The rule of " $50 \%$ relevance" refers to the correlation degree of cloud droplets located at the level boundary being equal to that of adjacent levels; both are $50 \%$. By using the rule, an extension cloud with a blurred boundary at adjacent levels is obtained, which indicates that the critical value belongs to both the upper and lower levels. The calculation formula is as shown in

$$
E_{n}^{\prime \prime}=\frac{c_{\max }-c_{\min }}{2.355} \text {. }
$$

3.3.3. Cloud Entropy Optimization Algorithms. These two methods determined the entropy value of the graded normal cloud model from different angles, which may lead to conflicting state determination conclusions [21, 43]. Therefore, a cloud entropy optimization algorithm is used to solve the entropy problem in this study, which guarantees the clarity of the classification and takes into account the principle of fuzziness.

Assuming that the actual measurement data of an evaluation index are represented by $x_{i}$ and its state level is $m$, the corresponding m-group hierarchical cloud model is established. $\left(E_{x}^{(j)}\right)_{1 \times m}$ and $\left(H_{e}^{(j)}\right)_{1 \times m}$ represent the cloud expectation set and cloud super-entropy set, respectively, $\left(E_{n^{\prime}}^{(j)}\right)_{1 \times m}$ and $\left(E_{n}^{(j)}\right)_{1 \times m}$ are the cloud entropy sets calculated by the above two algorithms, $\left(E_{n}^{(j)}\right)_{1 \times m}$ is the optimal cloud entropy set obtained by combinatorial optimization, and $j(j=1,2, \ldots, m)$ represents the rank number.

From the cloud correlation degree, function $u(x)$ can be regarded as a monotonically increasing function with $E_{n^{\prime}}$ as its independent variable, so for $E_{n^{\prime}}^{(j)}<E_{n}^{(j)}$, the minimum correlation degree of corresponding grade $j$ is the value of $u(x)^{\prime(j)}$ under the " $3 E_{n}$ " rule algorithm, expressed as $u(x)_{\min }^{\prime \prime}$, and the maximum correlation degree of 
corresponding grade $j$ is the value of $u(x)^{\prime \prime(j)}$ under the " $50 \%$ relevance" rule algorithm, expressed in $u(x)_{\max }^{\prime \prime}$. Let the cloud entropy optimization algorithm get the correlation degree of grade $j$ asu $(x)^{(j)}$, and use $\Delta u(x)_{\max }^{(j)}$ to express the maximum deviation of grade $j$ correlation degree. The constructed function is shown in

$$
\begin{aligned}
\Delta u(x)_{\max }^{(j)}= & \left(\Delta u(x)_{\max }^{\prime \prime(j)}-\Delta u(x)^{(j)}\right)^{2} \\
& +\left(\Delta u(x)^{(j)}-\Delta u(x)_{\max }^{\prime(j)}\right) .
\end{aligned}
$$

Then, according to the principle of minimizing the maximum deviation, the sum of the maximum correlation deviations of the indicator observations $x_{i}$ for the standard hierarchical cloud model of group $m$ should be minimized. Therefore, a nonlinear decision-making model of $\Delta u(x)_{\max }^{(j)}$ can be constructed, which is shown in

$$
\begin{cases}\min \Delta u(x)_{\max } & \left(E_{n}\right)=\sum_{j=1}^{J} \Delta u(x)_{\max }^{(j)} \\ \text { s.t. } & E_{n^{\prime}}^{(j)} \leq E_{n}^{(j)} \leq E_{n_{n}}^{(j)} .\end{cases}
$$

Based on the above model, the optimal cloud entropy set $E_{n}=\left(E_{n}^{(j)} 1 \times m\right)$ of the industry risk is obtained.

\subsection{Risk-Assessment Method Based on Entropy Weight-Cloud} Model. In order to make the results of the new energy vehicle industry risk assessment more objective and accurate, the entropy weight method and the cloud model were combined to evaluate and quantify the industry risk. The entropy weight method was used to determine the weight of each risk-evaluation index system, and the one-dimensional normal cloud model and synthesized cloud generator are used to evaluate industry risk. Figure 4 shows a detailed evaluation process consisting of three stages.

Stage 1: construct of the industry risk assessment index system.

This paper analyzes industry-risk-formation mechanism from both an endogenous and an exogenous perspective and then combines the expert analysis method and fuzzy statistical method to screen out the endogenous and exogenous risk evaluation factors. On the basis of the above in-depth analysis and digging, the industry-risk-assessment index system is constructed.

Stage 2: determine the weight of each risk index on the basis of the entropy weight method.

It investigates the importance of each risk factor through the expert-survey method and determines the weight of each indicator on the basis of the entropy weight method.

Stage 3: evaluate the risk of the new energy automobile industry in China's Jiangsu province in July 2019 on the basis of the cloud model. Then, propose industry-riskpreventing suggestions on the basis of the evaluation results.
In this stage, the first step is to classify the industry risk level. Next, it constructs the membership function of the cloud model and uses the three eigenvalues of the cloud model to reflect the membership relationship between the risk factors and the risk levels. Then the forward cloud generator is used to perform a series of calculations on the cloud model to obtain the cloud drop distribution of each of the secondary risk factors. Combined with the weight of each risk factor, the study uses the integrated cloud generator to calculate the characteristic values of the main risk factors and comprehensive industry risk obtaining the cloud drop distribution of each risk. Finally, based on the evaluation results, industry risk method measures are proposed.

\section{Results and Discussion}

\subsection{Construct the Risk-Assessment Index System}

\subsubsection{Analysis of Industry-Risk-Formation Mechanism}

(1) Analysis of the Formation Mechanism of Endogenous Risk. The new energy automobile industry is compared to the human body in this study. The innate quality of the body is like the foundation of industrial development; if innate quality is good, the ability of the industry to withstand risk is strong. The proportion of human-body function elements is like the industrial structure; if production factors are with reasonable composition and good coordination, the ability of the industry to withstand risk is strong. The network can be understood as the interactive relationship of industry production components. The more coordinated the various entities are, the stronger the industry's ability to resist risks. Therefore, endogenous risk was divided into fundamental, structural, and network risk [44].

(1) Fundamental Risk. The industrial foundation is the basic status of industrial development. The development of an industry is directly related to the industrial foundation of a country or region. Fundamental risk is caused by different basic conditions of the production factors during the new energy automobile industry. According to the theory of production factors, if the industry has good innate factors of production, the industry's ability to resist risks is strong; otherwise, the ability is weak.

The industrial foundation is closely related to innate factors and practical capabilities of industrial development. Innate factors are factors directly related to innate endowments and reflect the industry's possible innate qualities and growth potential. They include resource endowment, research and development technology level and reserves, the strength of obtained financial support, the quality of labor force, and the management level of enterprises. If the innate factors of the industry are weak, the possibility of the industry forming fundamental risks is greater. The real ability is influenced by innate factors, and it is a series of capabilities shown in the industrialdevelopment process, such as technological- 


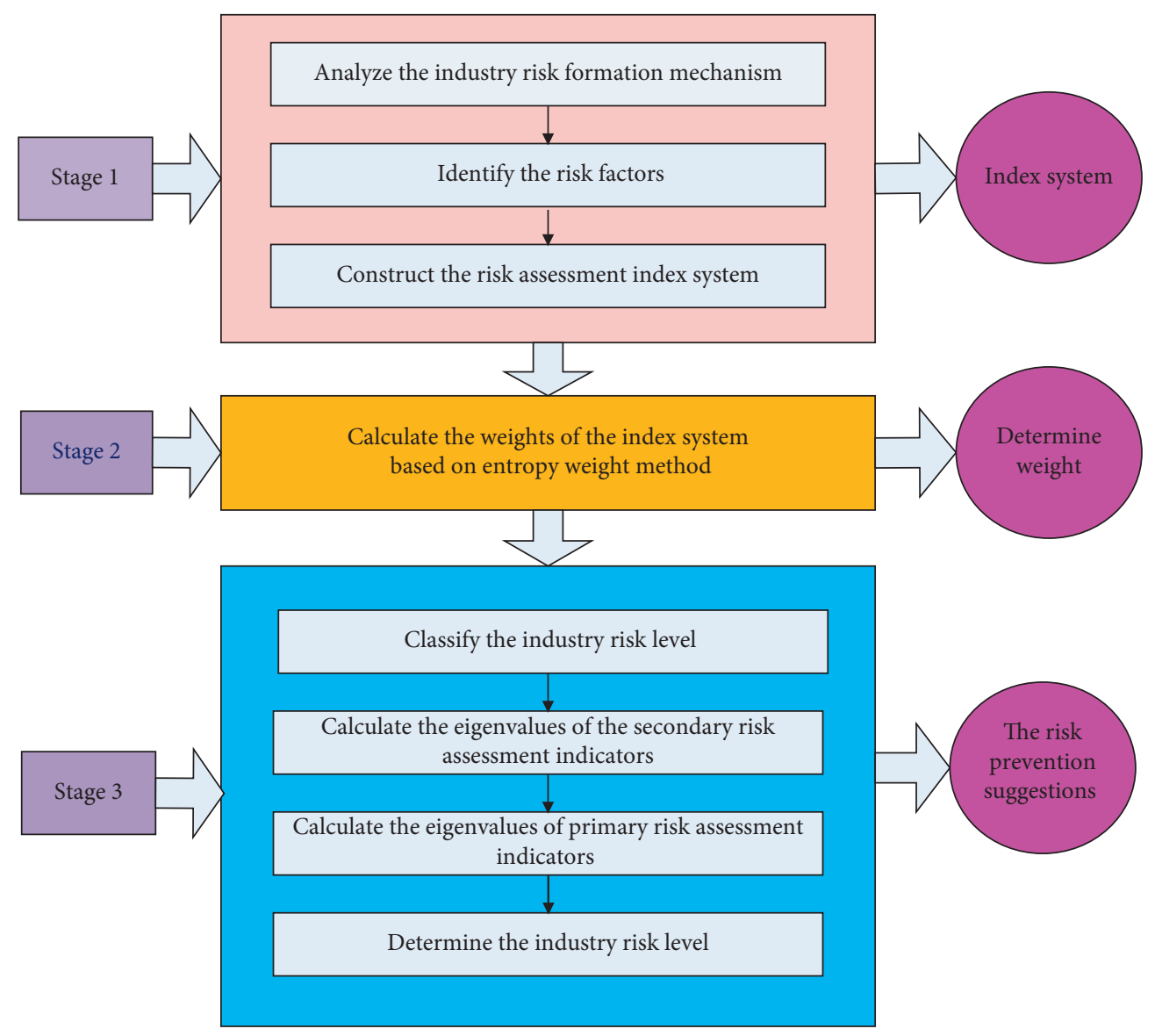

FIgURE 4: Evaluation process consisting of three stages.

innovation, financing, marketing, and production capabilities. These capabilities are directly related to the development status of the new energy automobile industry. If real industrial capacity is weak, the possibility of industry risk is also greater.

Real ability is affected by the innate factors of the industry, and it has a more direct relationship with its own development. If the innate endowment of the new energy vehicle industry is insufficient, support for the industry's actual capabilities may be insufficient, so it is easy to form fundamental risk. However, even if the industry's innate factors are similar, if the industry's own actual development status is different, it also leads to differences in real capabilities, then forming fundamental risk. The mechanism of fundamental risk is shown in Figure 5.

(2) Structural Risk. This risk may be caused by structural factors such as the composition of different scales of enterprises, the composition of raw materials, the composition of the industrial chain, and regional distribution in the industry when the industry is statically observed. If state-owned enterprises in the region are too strong, this easily leads to a monopoly, which affects the enthusiasm of private enterprises for innovation. If the region lacks core enterprises, the market concentration is low; this may lead to excessive resources and weak industrial competitiveness. The battery is the core of new energy vehicles. At present, raw materials for producing batteries are mainly lithium, cobalt, and manganese. As the number of new energy vehicles increases, the competition for global metal resources becomes more intense. However, the main power-battery resources such as lithium metal and cobalt in Jiangsu are lacking. Therefore, the stable supply of new energy vehicle resources and price stability are facing greater challenges. In view of the limited types of raw materials for the production of batteries, the entire new energy automobile industry chain is constructed on the basis of these metal resources. The product variety is single. Once metal resources cannot be supplied in time, the entire industrial chain faces the risk of being replaced. The new energy automobile industry should be rationally distributed according to factors such as regional industrial-development basis, market-distribution characteristics, and trade facilitation. For example, if the government develops the new energy automobile industry too blindly, it may lead to industrial homogenization and fragmentation. The formation mechanism of structural risk is shown in Figure 6. 


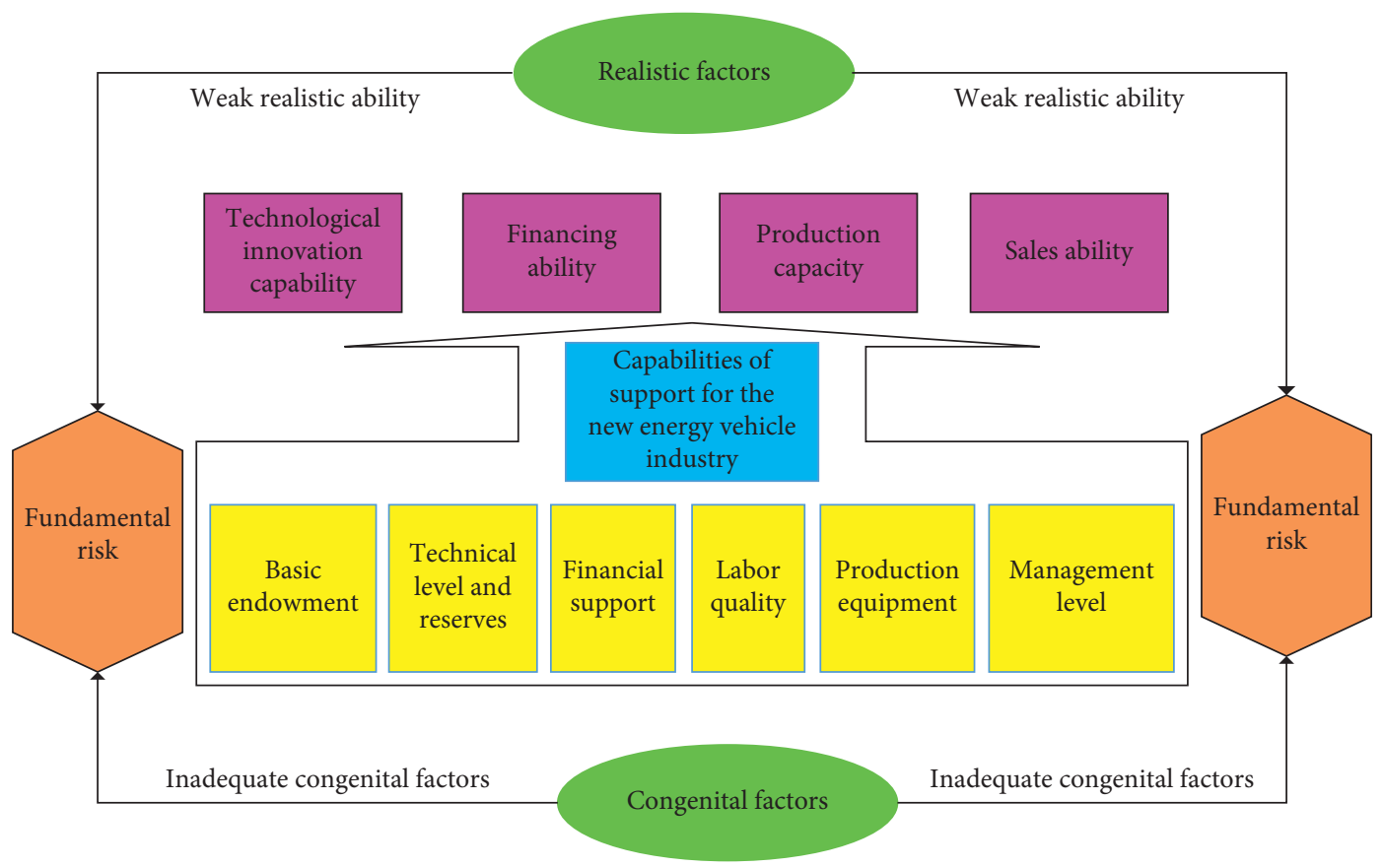

FIgURE 5: Analysis of the formation mechanism of fundamental risk.

(3) Network Risk. According to economic and socialnetwork theory, the new energy automobile industry can be regarded as a network composed of subjects, resources, and behaviors. Network risk occurs in the early stage of the development of the new energy automobile industry. During interactions between subject and resource, due to a lack of capital, technology, information, talents, and material resources, there are some harmful behaviors appearing in the industry, such as unfair competition, excessive dependence on policies, incomplete contracts, and moral hazards. These behaviors affect the development of the industry.

As shown in Table 1, the main body of the new energy automobile industry mainly includes consumers, vehicle manufacturers, supporting manufacturers, scientific research institutions, governments, and operation and maintenance companies. Resources include basic information and policy resources. Behaviors occur between subjects such as behavioral trading, competition, cooperation, regulation, and research and development. Interactions between subjects form various relationships, such as competition, cooperation, information, and competition and cooperation.

In the process of interaction between subject and resources, relevant subjects play a game with each other, and there are some behaviors that adversely affect the industry, such as unfair competition, excessive dependence on policies, and incomplete fulfillment of contracts and moral hazards. These actions form network risks of the industry. The formation mechanism of structural risk is depicted in Figure 7.

(2) Analysis of the Formation Mechanism of Exogenous Risk. Exogenous risk comes from outside the industry. It originates from the complexity and uncertainty of the environment in which the new energy automobile industry exists, such as the regional ecological environment, economic cycles, external competition, and policy or legal risks. Exogenous risk has the characteristics of wide range, contingency, and suddenness. Compared with endogenous risks, exogenous risk is more difficult to control. The external factors of the industry itself cannot create risks, but changes in external factors change the industry's relatively stable internal environment, thus impacting the industry and leading to risk.

The perfect policy system is conducive to the development of the new energy automobile industry, or it would bring great risk to the industry. The development of the new energy automobile industry has a relatively significant government-driven situation, which probably cause excessive government intervention in the market and affect the effective allocation of resources. Therefore, the new energy auto industry faces policy risk. Environmental change itself does not create risk. Environmental factors act on the industry, and exerting influence on the industry can lead to risk. For example, low-economic, imperfect-legal, fragilezonal-ecological, and poor recycling, destruction and storage environments of power batteries affect the development of new energy vehicles, thus making the industry face greater environmental risk.

The market factor is also important, affecting the development of new energy vehicles. The development of new energy vehicles not only faces the competitive pressure of traditional-fuel vehicles, but also faces the competitive pressure of foreign new energy vehicles. At present, compared with traditional-fuel vehicles, Jiangsu's new energy vehicles have no obvious competitive advantage, and there is still a big gap with internationally advanced new energy vehicles. Moreover, society perceptions of new energy 


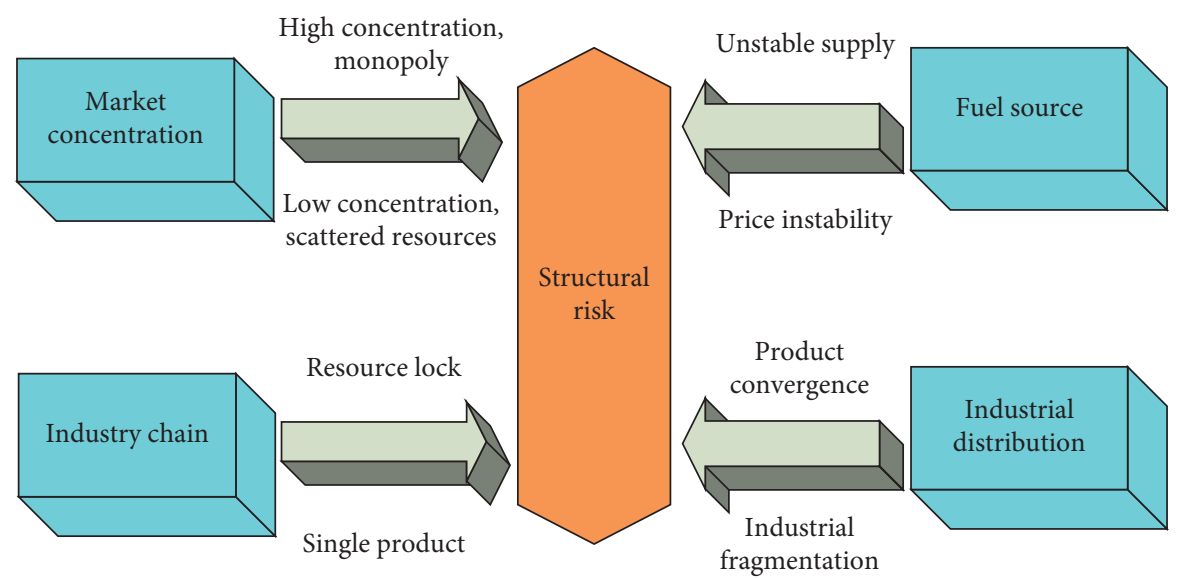

FIgURE 6: Analysis of the formation mechanism of structural risk.

TABle 1: Main contents of subjects, resources, behavior, and relationship of new energy automobile industry.

\begin{tabular}{|c|c|}
\hline Subjects & $\begin{array}{c}\text { Consumers, vehicle manufacturers, supporting manufacturers, research institutions, governments, battery operators, and } \\
\text { charging maintainers }\end{array}$ \\
\hline Resources & Basic resources, information resources, policy resources \\
\hline Behavior & Buying and selling, competition, cooperation, regulation, research, and development \\
\hline Relationship & Competition, cooperation, information, competition and cooperation, policy \\
\hline
\end{tabular}

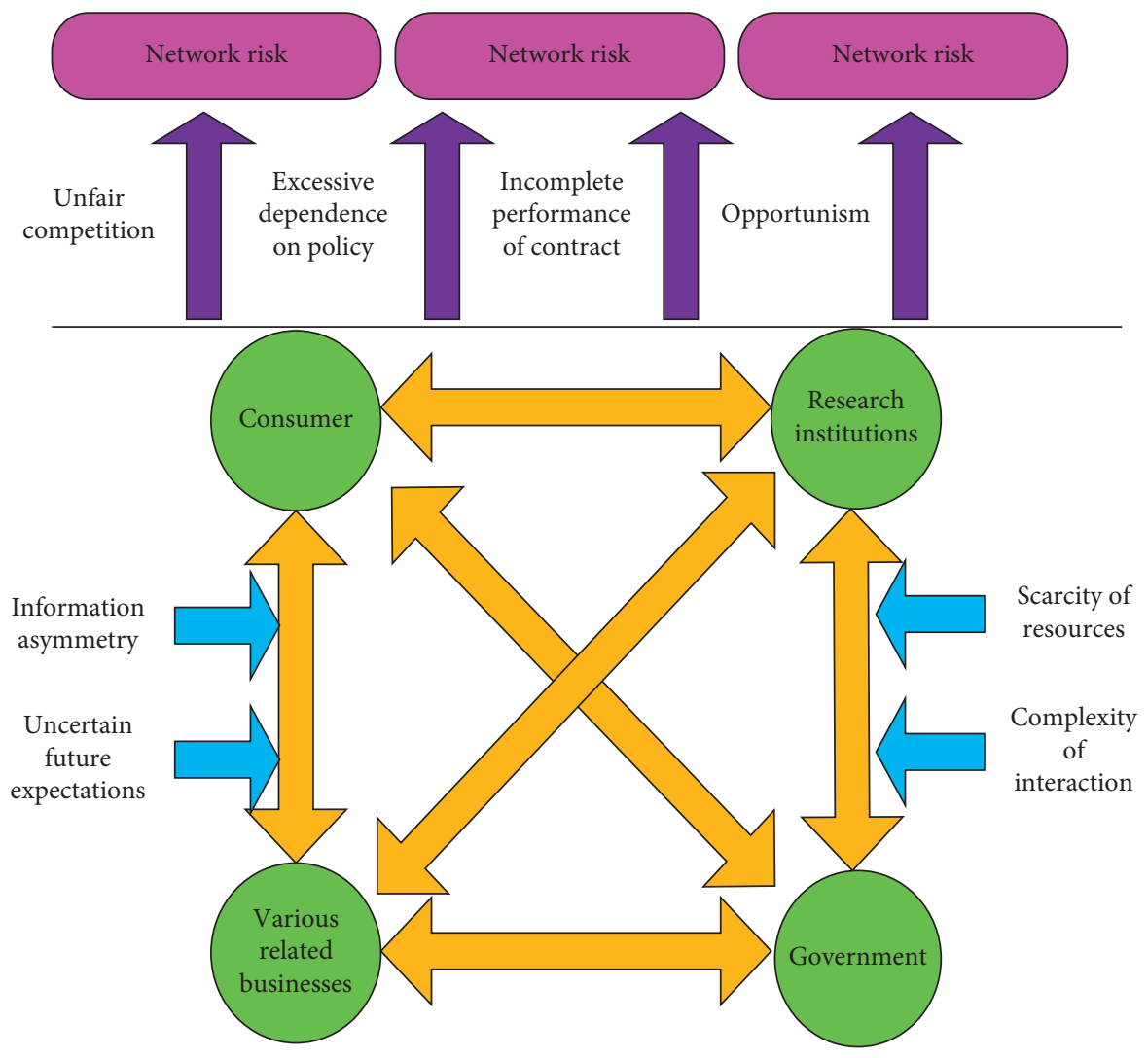

Figure 7: Analysis of the formation mechanism of network risk.

vehicles are still shallow, and consumption patterns have not changed; low-carbon and -energy consumption habits have especially not yet been developed, and insufficient high-end capacity and low-end overcapacity coexist. All of these affect the development of new energy industries, thereby leading to market risk during the industry. 
There is a mutually reinforcing mechanism between endogenous and exogenous risks (Figure 8). If the endogenous risk is large, the industry's ability to resist risk is also weak, which leads to great impact on exogenous risk. Once the exogenous risk is formed, the relative steady state of endogenous factors is broken, which increases the possibility of endogenous risk.

4.1.2. Pathways of Identifying the Risk Factors. Combining the above analysis of the industrial formation mechanism, three pathways were used to identify the risk factors of new energy automobile industry: (1) reading the domestic and foreign literature related to the new energy automobile industry; (2) conducting consultations, exchanges, and interviews with stakeholders involved in new energy automobile industry to obtain relevant risk factors; and (3) comparing the situation of other industry risk types and taking them as reference to determine risk factors. After modification for several times in this paper, the risk of the new energy automobile industry was divided into endogenous and exogenous risks on the basis of endogenous and exogenous perspectives. Endogenous risk refers to the risk faced by the industry itself, and exogenous risk refers to risk from the external environment. Endogenous risk is the industry risk caused by internal causes and can reflect the industrial quality. Exogenous risk originates from the complexity and uncertainty of the environment in which the new energy automobile industry exists.

\subsubsection{Constructing Risk-Assessment Index System.}

Through reading the domestic and foreign literature related to the new energy automobile industry, this paper analyzed the risk-formation mechanism from endogenous and exogenous perspectives and then constructed the primary indicators. Endogenous risk was divided into fundamental, structural, and network risks. Exogenous risk was subdivided into policy, market, and environmental risks. Then, layer by layer, a hierarchical structure including six primary indicators and twenty secondary indicators was formed. Among second level indicators, fuel-source, unfair-competition, incomplete-contract, moral, excessive-policy-dependence, social-cognitive, and overcapacity risks were identified through exchanging and interviewing stakeholders involved in the new energy automobile industry; regional-distribution, industrial-chain, international-competition, industry-transformation, and zonal-ecological risks were identified through comparing the situation of other industry risk types [45-48] and taking them as reference to determine risk factors. Other secondary indicators were identified from reading the domestic and foreign literature related to the new energy automobile industry $[49,50]$. The risk-evaluation index system is shown in Table 2 .

The definition and interpretation of primary indicators were introduced in the analysis of the industrial-risk formation mechanism. The definition and interpretation of secondary risk-assessment indicators are explained as follows.
(1) Fundamental Risk. Fundamental risk mainly includes technological-innovation, financing-ability, production-efficiency, and production-cost risks. The possibility that the lack of technological innovation capability, high cost of the product, low production efficiency, and financing difficulties affect industrial development forms the four risks above, respectively.

(2) Structural Risk. Structural risk mainly includes market-concentration, fuel-source, regional-distribution, and industrial-chain risks. The lack of core enterprises in the industry, resulting in excessively dispersed resources and low market concentration, which brings risk to the development of the industry, forms the market-concentration risk. Fuel-source risk is the risk that the industry is limited to a certain resource due to restrictions of fuel supply. Industrialchain risk is the risk caused by the unreasonable structure of the new energy automobile industry chain. Regional-distribution risk is the possibility that unreasonable regional distribution adversely affects the development of the industry.

(3) Network Risk. Network risk mainly includes unfaircompetition, incomplete-contract, moral-hazard, and excessive-policy-dependence risk. Risk caused by unfair competition and incomplete contracts among various stakeholders are called unfair-competition and incomplete-contract risks, respectively. Due to information asymmetry, subjects with competing relationships, such as research institutions, maximize their own utility while engaging in behaviors that are not conducive to the actions of others. This possibility of having impact on the industry is a moral risk. The risk of excessive dependence on policies refers to the possibility that the new energy automobile industry is overreliant on government support policies, thereby affecting the development of the industry.

(4) Policy Risk. Policy risk mainly includes policy-stability, -continuity, -timeliness, and -coordination risks. Unstable, discontinuous, untimely, and uncoordinated policies on the industry form the four secondary riskevaluation indicators above, respectively.

(5) Market Risk. Market risk mainly includes international-competition, industrial-transformation, society-cognitive, and overcapacity risks. The development of new energy automobiles faces market competition in foreign new energy automobiles. The possibility of this international competitive disadvantage affecting the development of the industry is the risk of international competitiveness. It is a transformation from traditional cars to new energy vehicles. Industry-transformation risk is the possibility of social impact on the industry development with the reconversion of work. At present, society has low awareness of new energy automobiles and cannot accept such automobiles, 


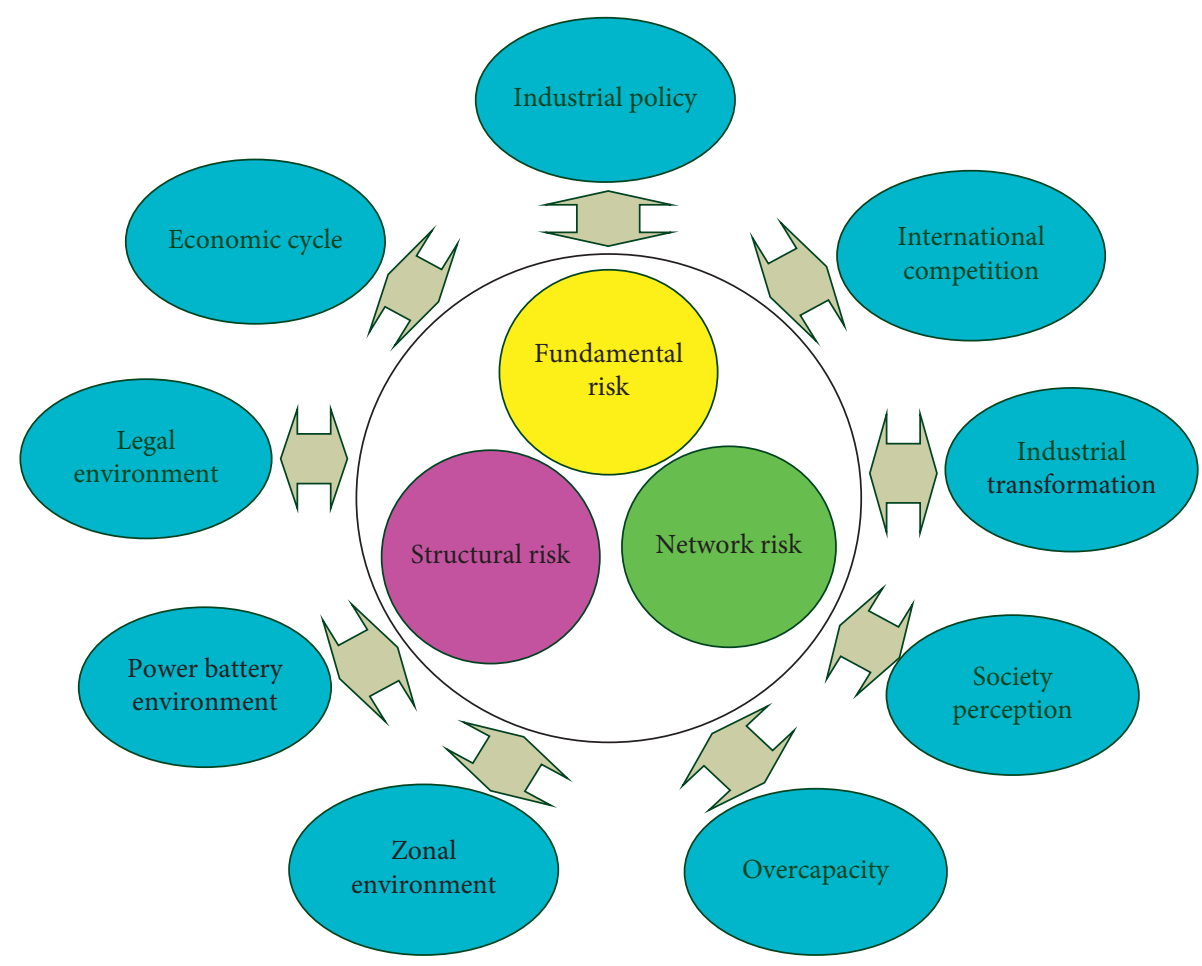

Figure 8: Analysis of the formation mechanism of exogenous risk.

TABLE 2: Risk-evaluation index system of new energy automobile industry in China's Jiangsu province.

\begin{tabular}{lcc}
\hline Risk perspective & $\begin{array}{c}\text { Primary-indicator-factor } \\
\text { layer }\end{array}$ & Secondary-indicator-factor layer \\
$\begin{array}{l}\text { Endogenous } \\
\text { risk }\end{array}$ & $\begin{array}{c}\text { Fundamental risk } \\
\text { Structural risk } \\
\text { Network risk }\end{array}$ & $\begin{array}{c}\text { Technological-innovation, production-cost, production-efficiency (output value), and } \\
\text { financing-ability risks }\end{array}$ \\
& Policy risk & $\begin{array}{c}\text { Market-concentration, fuel-source, regional-distribution, and industrial-chain risks } \\
\text { Unfair-competition, incomplete-contract, moral, excessive-policy-dependence risks }\end{array}$ \\
Exogenous risk & Market risk & $\begin{array}{c}\text { Policy-stability, -continuity, -timeliness, and -coordination risks } \\
\text { International-competition, industry-transformation, social-cognitive, and overcapacity } \\
\text { risks }\end{array}$ \\
& Environmental risk & $\begin{array}{c}\text { Legal-environment, power-battery-environment, economic-cycle, and zonal-ecological } \\
\text { risks }\end{array}$ \\
\hline
\end{tabular}

forming a societal-perception risk. The new energy automobile industry has experienced blind expansion and overheated investment, which may increase the overall overcapacity risk of automobiles and power batteries.

(6) Environmental Risk. Environmental risk mainly includes legal-environment, power-battery-environment, economic-cycle, and zonal-ecological risks. Legal-environmental risk is the possibility that the uncertainty of the legal environment in which the new energy vehicle industry is located affects the development of the industry. The possibility that poor recycling, destruction, and storage of discarded batteries affect industrial development forms the battery-environment risk. Business cycle is also an important factor affecting industrial environment. Changes in economic cycle caused by sudden, uncontrollable, and cyclical fluctuations in the external economy have an impact on industry, thereby forming the economic-cycle risk. Zonal-ecological risk mainly refers to the impact of environmental pollution, human activities, and natural disasters on the industrial ecological environment, local and external.

4.2. Classification of Risk Levels. Risk is an uncertain description of future results. Most scholars use qualitative language to define risk level. It is generally sorted into "low," "lower medium," "medium," "higher medium", and "high" risk. The description of risk level is the lack of objectivity of quantitative data. Some of the literature uses weights for risk measurements. Even though this method can quantify risk, once weights are determined, the risk loses randomness and ambiguity [51]. The cloud model was applied to classify and evaluate risk in this study, which achieved the conversion of 
a qualitative concept into quantitative concept and reduced loses of information during the period of conversion. Moreover, numbers in the range from 0 to 5 were used to measure risk. A small value indicated low risk, and vice versa. In order to calculate the expectation, entropy, and super entropy of each level of risk, ten experts in the new energy industry were invited to assign values for low, lower medium, medium, higher medium, and high risk in July 2019 (as shown in Table 3). This study used the forward cloud generator in the cloud model to generate a risk-level cloud map (Figure 9).

The horizontal axis represents the domain of evaluation scores, and the vertical axis represents the membership degree. Each cloud droplet has its corresponding eigenvalues $\left(E_{x}, E_{n}, H_{e}\right)$. These green, blue, yellow, red, and purple cloud droplets represent the distribution of the graded normal cloud model of low-high risk, respectively.

4.3. Determination of Indicator Weight. The influence degrees of each first and second level index on the risk of the new energy automobile industry were different, so it was necessary to determine the degree of influence of each risk factor. The risk-factor weight of the new energy automobile industry in China's Jiangsu province is shown in Table 4. In addition, the weight of endogenous and exogenous risks was 0.4111 and 0.5889 , respectively, based on the entropy weight method shown in Table 4. Obviously, the impact of exogenous risk on the new energy vehicle industry was greater than that of endogenous risk.

4.4. Industry Risk Assessment Based on the Cloud Model. Ten experts in the field of new energy automobile industry in Jiangsu were invited to score each risk and analyze the scores in July 2019. Take fundamental risk, for example, which includes technological-innovation, production-cost, production-efficiency, and financing-capacity risks. The expectation $\left(E_{x}\right)$ and entropy $\left(E_{n}\right)$ of each risk factor were calculated in this study.

$$
E_{x}=\frac{\left(E_{x 1}+E_{x 2}+\cdots+E_{x n}\right)}{n}
$$

where $E_{x}$ is the expectation of a risk factor. $E_{x 1}, E_{x 2}, \ldots, E_{x n}$ are the expected values calculated based on the expert scores for every risk factor according to (12) and $n$ represents the number of experts.

Entropy value solution based on the cloud entropy optimization algorithm:

$$
H_{e}=k \text {, }
$$

where $H_{e}$ is the hyper entropy of a risk factor, which reflects the randomness and ambiguity of the evaluation, and $k$ is a constant, of which the value is adjusted according to the fuzzy threshold of risk concentration factors. If the hyper entropy result is too large, the uncertainty of the evaluation is increased; therefore, it was assigned a value of 0.1 in this article. This can not only objectively reflect the randomness and ambiguity of risk assessment, but also simplify the evaluation process. Calculation results of the three eigenvalues included in the subrisk factor are shown in Table 5.

Four subrisk factors all affect the main factor risk, that is, fundamental risk; a one-dimensional integrated cloud could be used to represent it. Using equation (17), (18), and the cloud entropy optimization algorithm, this study calculated three eigenvalues of fundamental risk. Using the same principle, three eigenvalues of five other main factor risks, namely, structural, network, policy, market, and environmental risks, were calculated as shown in Table 6 .

$$
\begin{aligned}
& E_{x}=\frac{E_{x 1} E_{n 1} W_{1}+E_{x 2} E_{n 2} W_{2}+\cdots+E_{x n} E_{n n} W_{n}}{E_{n 1} W_{1}+E_{n 2} W_{2}+\cdots+E_{n n} W_{n}}, \\
& E_{n}=E_{n 1} W_{1}+E_{n 2} W_{2}+\cdots E_{n n} W_{n} .
\end{aligned}
$$

In (19), expectations for a risk: $E_{n 1}, E_{n 2}, \ldots, E_{n n}$ represent the $n$-th expert score for the risk. $E_{n 1}, E_{n 2}, \ldots, E_{n n}$ are the entropy of a risk factor. In (20), $W_{1}, W_{2}, \ldots, W_{n}$ represents the weight of a risk.

We can calculate the expectation, entropy, and hyper entropy of the comprehensive risk using (19) and (20), the expectation $\left(E_{x}\right)$ was equal to 2.8849 , and entropy $\left(E_{n}\right)$ was equal to 0.9500 . Expectation $\left(E_{x}\right)$ affects the position of the center of gravity of the cloud drop, indicating the average value of the risk-factor membership; entropy $\left(E_{n}\right)$ describes the ambiguity and randomness of the cloud drop, reflecting the possible range of risk-factor weights and membership degrees; hyper entropy is the entropy of entropy, which describes the thickness of the cloud and reflects the degree of dispersion of the cloud droplets, indicating the proportion of the various risk factors and the deviation of the membership from the average. From the three eigenvalues of each risk, the cloud model map of the main factors and the comprehensive risk comparison cloud model map could be obtained by using MATLAB software, as shown in Figures 10-12.

Figure 10 shows the cloud map of endogenous risk, Figure 11 shows the cloud map of exogenous risk, and Figure 12 shows the cloud map of new energy automobile industry risk in Jiangsu. In the radar chart shown in Figure 13, red lines represent the weight of six primary risk factors indicators; that is, the importance of primary risk factors is represented by red lines, and the importance of dotted lines increases from inside to outside. Blue lines represent the risk level of the six primary-risk-factor indicators, and the grade is higher as you move from inside to outside.

\subsection{Evaluation-Result Analysis}

4.5.1. Endogenous Risk Analysis. Figure 10 shows that the expectations of fundamental, structural, and network risks were $3.2028,2.6287$, and 2.7321 , respectively. The cloud map of fundamental risk was basically consistent with the higher medium risk; therefore, fundamental risk was a higher medium risk. The cloud maps of structural and network risks were between medium and higher medium risk, and structural and network risks were moderately high medium 
TABLE 3: Eigenvalues table for different risk levels of cloud models.

\begin{tabular}{lccc}
\hline Risk level & Expectation $\left(E_{x}\right)$ & Entropy $\left(E_{n}\right)$ & Hyper entropy $\left(H_{e}\right)$ \\
\hline Low risk & 0.26 & 0.50 & 0.1 \\
Lower medium risk & 1.23 & 0.62 & 0.1 \\
Medium risk & 2.29 & 0.69 & 0.1 \\
Higher medium risk & 2.906 & 0.78 & 0.1 \\
High risk & 3.9 & 0.81 & 0.1 \\
\hline
\end{tabular}

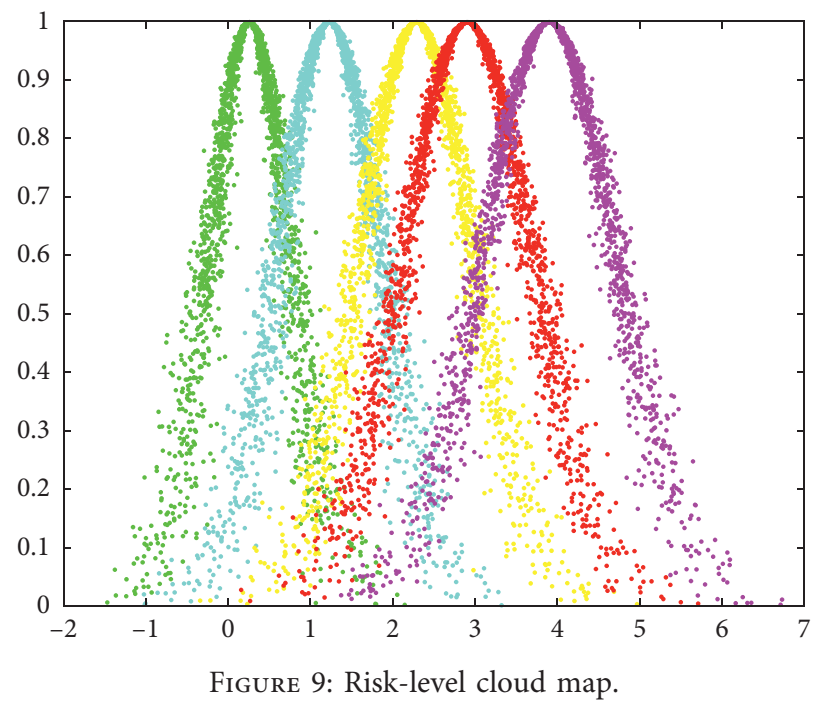

TABLE 4: Risk-factor weight of new energy automobile industry in China's Jiangsu province.

\begin{tabular}{|c|c|c|c|c|}
\hline $\begin{array}{l}\text { Risk indicator } \\
\text { Risk weight } \\
\end{array}$ & Primary-indicator-factor layer & Primary-indicator weight & Secondary-indicator-factor layer & $\begin{array}{c}\text { Secondary-indicator } \\
\text { weight }\end{array}$ \\
\hline \multirow{12}{*}{$\begin{array}{l}\text { Endogenous risk } \\
(0.4111)\end{array}$} & \multirow{4}{*}{ Fundamental risk } & \multirow{4}{*}{0.1666} & Technological-innovation risk & 0.3975 \\
\hline & & & Production-cost risk & 0.2000 \\
\hline & & & $\begin{array}{c}\text { Production-efficiency risk (output } \\
\text { value) }\end{array}$ & 0.2224 \\
\hline & & & Financing-ability risk & 0.1801 \\
\hline & \multirow{5}{*}{ Structural risk } & \multirow{4}{*}{0.1074} & Fuel-source risk & 0.2091 \\
\hline & & & Market-concentration risk & 0.3277 \\
\hline & & & Regional-distribution risk & 0.2583 \\
\hline & & & Industrial-chain risk & 0.2049 \\
\hline & & \multirow{4}{*}{0.1371} & Unfair-competition risk & 0.1423 \\
\hline & \multirow{3}{*}{ Network risk } & & Incomplete-contract hazard & 0.2302 \\
\hline & & & Moral risk & 0.4092 \\
\hline & & & Risk of excessive policy dependence & 0.2183 \\
\hline \multirow{12}{*}{$\begin{array}{l}\text { Exogenous risk } \\
(0.5889)\end{array}$} & \multirow{4}{*}{ Policy risk } & \multirow{4}{*}{0.1582} & Policy-stability risk & 0.2000 \\
\hline & & & Policy-continuity risk & 0.3773 \\
\hline & & & Policy-timeliness risk & 0.2107 \\
\hline & & & Policy-coordination risk & 0.2120 \\
\hline & \multirow{4}{*}{ Market risk } & \multirow{4}{*}{0.1732} & International-competition risk & 0.1998 \\
\hline & & & Industry-transformation risk & 0.3014 \\
\hline & & & Social-cognitive risk & 0.3387 \\
\hline & & & Overcapacity risk & 0.1601 \\
\hline & \multirow{4}{*}{ Environmental risk } & \multirow{4}{*}{0.2575} & Legal-environment risk & 0.2188 \\
\hline & & & Power-battery-environment risk & 0.3209 \\
\hline & & & Economic-cycle risk & 0.1517 \\
\hline & & & Zonal-ecological risk & 0.3086 \\
\hline
\end{tabular}


TABLE 5: Cloud model eigenvalues of secondary-risk factors.

\begin{tabular}{|c|c|c|c|c|}
\hline Secondary-risk factors & Expectation $\left(E_{x}\right)$ & Entropy $\left(E_{n}\right)$ & Hyper entropy $\left(H_{e}\right)$ & Weights \\
\hline Technological-innovation risk & 3.5000 & 0.8869 & 0.1 & 0.3975 \\
\hline Production-cost risk & 3.3000 & 1.1826 & 0.1 & 0.2001 \\
\hline Production-efficiency risk (output value) & 2.8000 & 0.8869 & 0.1 & 0.2224 \\
\hline Financing-ability risk & 2.8000 & 0.5913 & 0.1 & 0.1801 \\
\hline
\end{tabular}

TABLE 6: Cloud model eigenvalues of primary-risk factors.

\begin{tabular}{lccccr}
\hline Primary-risk factors & $E_{x}$ & $E_{n}$ & $H_{e}$ & $W$ & $E_{x} \times E_{n} \times W$ \\
\hline Fundamental risk & 3.2028 & 0.8929 & 0.1 & 0.1666 & 0.4773 \\
Structural risk & 2.6287 & 0.8882 & 0.1 & 0.1074 & 0.2496 \\
Network risk & 2.7321 & 1.0195 & 0.1 & 0.1371 & 0.3831 \\
Policy risk & 2.9822 & 1.0608 & 0.1 & 0.1582 & 0.4863 \\
Market risk & 3.0084 & 0.8396 & 0.1 & 0.1732 & 0.4359 \\
Environmental risk & 2.8065 & 0.9818 & 0.1 & 0.2575 & 0.7084 \\
Total & & & & & 0.1678 \\
Comprehensive risk & 2.8849 & 0.9500 & & & 0.1454 \\
\hline
\end{tabular}

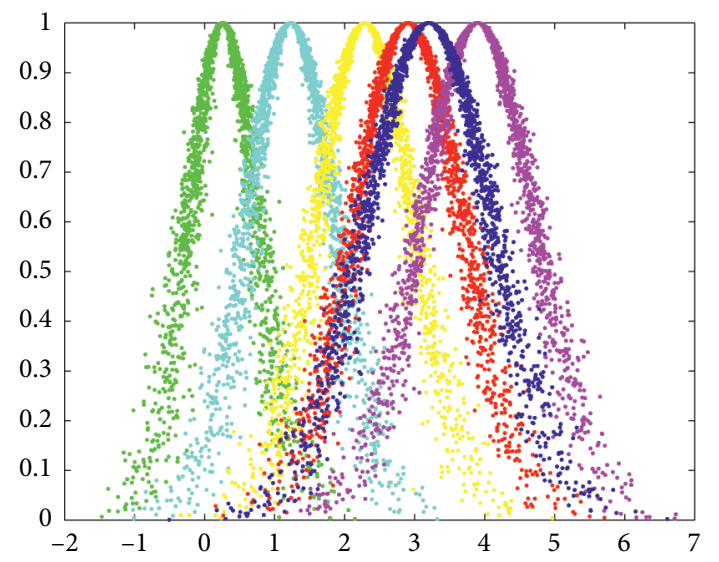

(a)

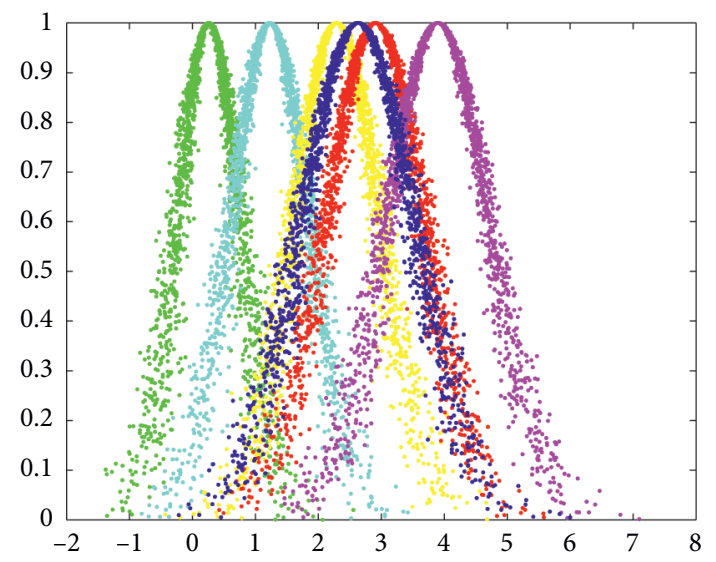

(b)

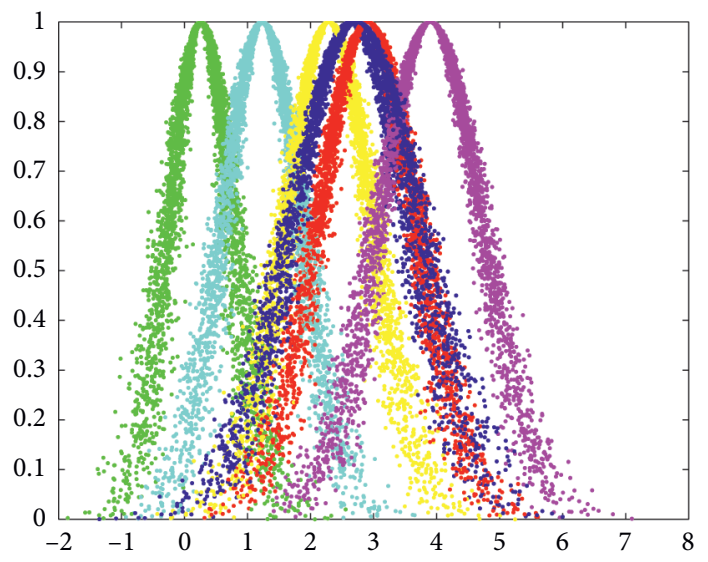

(c)

FIgURE 10: Cloud map of the endogenous risk. (a) Fundamental risk. (b) Structural risk. (c) Network risk.

risk, with the order of fundamental $>$ network $>$ structural risk. The technical element is the key factor to developing the new energy vehicle industry. However, the new energy vehicle industry in Jiangsu is facing higher technical risk. This is because a new energy vehicle power battery has not yet achieved a fundamental breakthrough, some key 


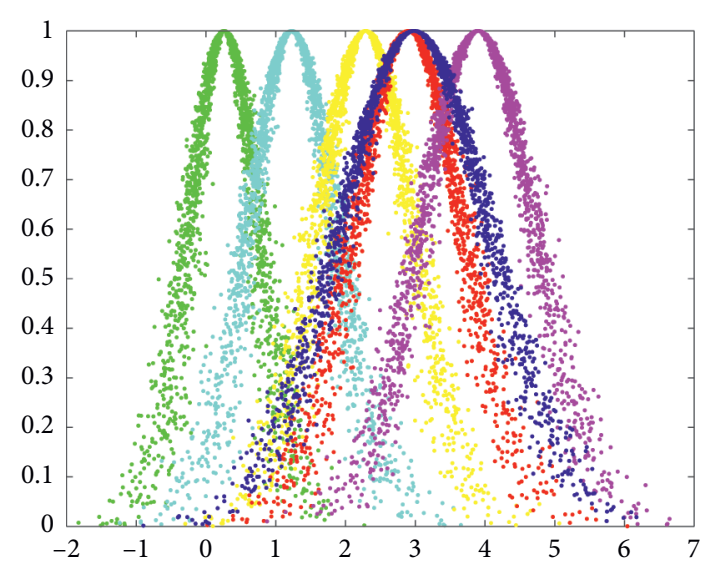

(a)

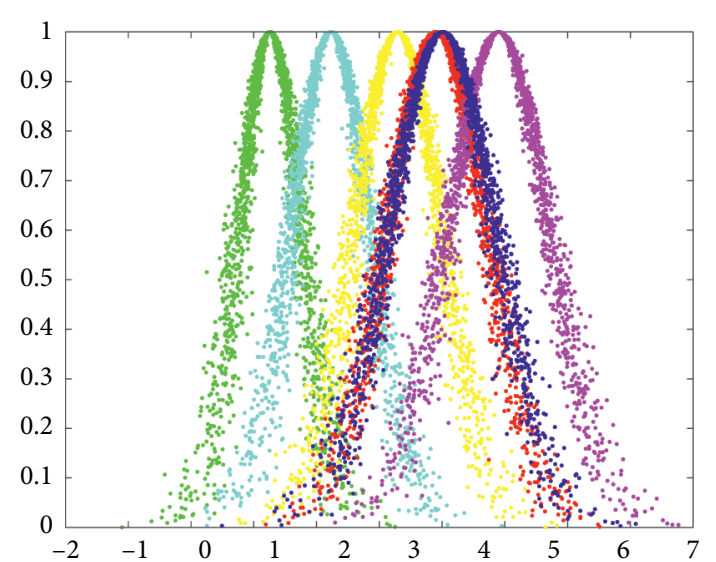

(b)

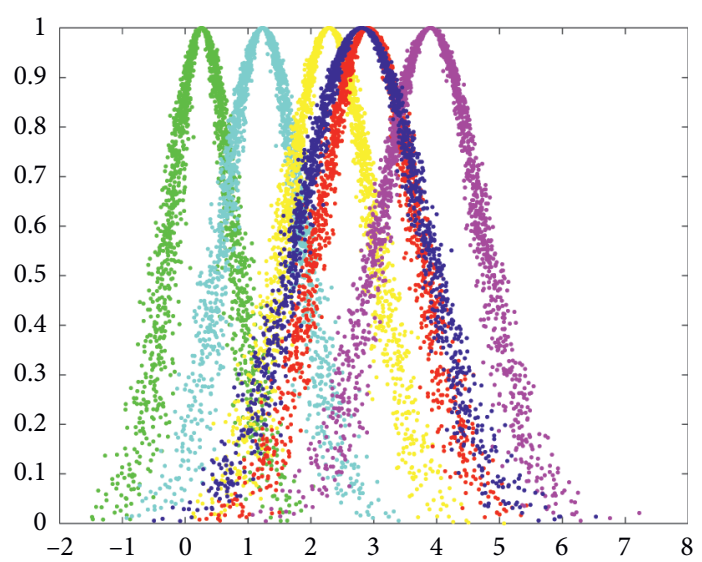

(c)

Figure 11: Cloud map of the exogenous risk. (a) Policy risk. (b) Market risk. (c) Environmental risk.

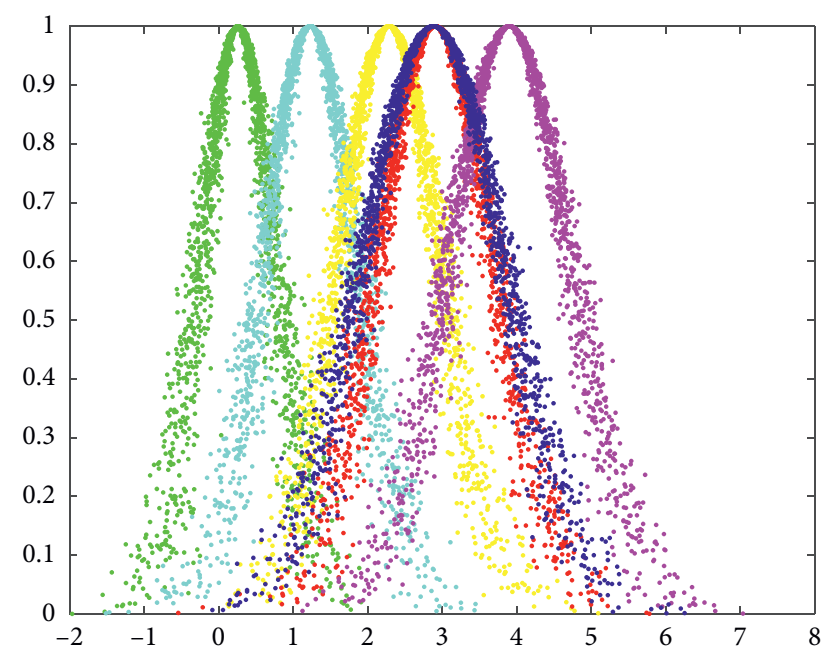

FIGURE 12: Cloud map of new energy automobile industry risk in Jiangsu province.

components still need to be purchased, the fuel-cell-vehicle industry chain lags behind, and a gap exists between Jiangsu province's product quality and that of advanced regions abroad. In addition, high product cost, low production efficiency, and financial constraints result in the industry

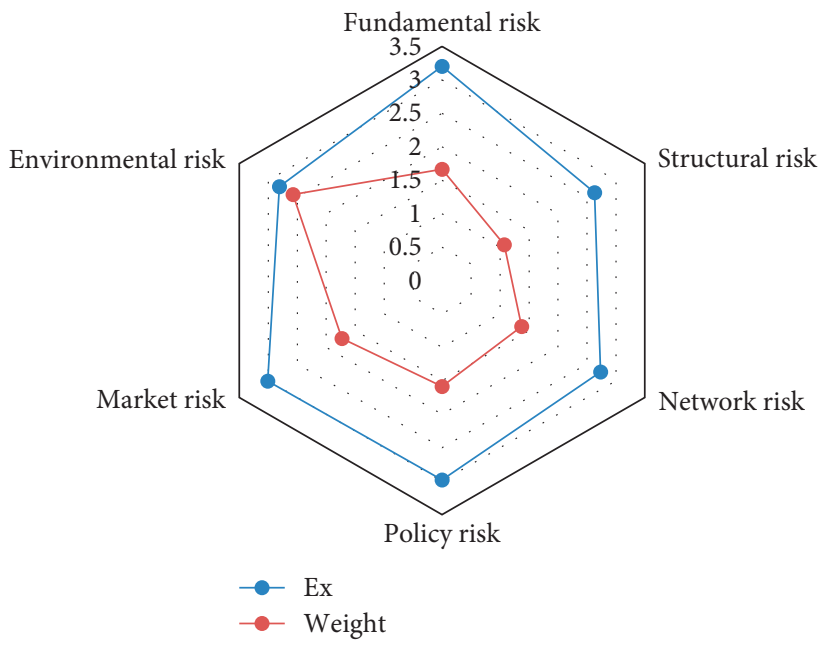

FIGURE 13: Level and weight of the primary risk indicator.

facing high production-cost, production-efficiency, and financing risks. On the basis of the above analysis, fundamental risk is a higher medium risk. Structural risk is at medium level and close to higher medium risk. First, although the core competitiveness of the new energy vehicle industry in Jiangsu has broken through, it is still not strong. 
Next, industry enterprises are widely distributed but not refined. Last but not least, resulting industry chains were created but are not perfect. On the basis of the above analysis, the main influencing factors of network risk were moral risk and the risk of overreliance on policies. Analysis showed that new energy vehicle companies in China's Jiangsu province overrely on policy support; therefore, the future market position is threatened by the other new energy vehicle companies outside the Jiangsu province. Additionally, unfair-competition, incomplete-contract, and moral risks are higher than the medium risk level, so the risk level of the whole network is higher than medium risk.

4.5.2. Exogenous Risk Analysis. As depicted in Figure 11, expectations of policy, market, and environmental risks were $2.9822,3.0084$, and 2.8065 , respectively. The cloud maps of policy and market risks were basically consistent with the higher-medium-risk cloud map. Therefore, policy and market risks were higher than medium risk, and the environmental-risk cloud map was located between the mediumand higher-medium-risk cloud map, which was very close to the higher-medium-risk cloud map, so environmental risk was moderately medium risk. Moreover, the order of external generating risks was market $>$ policy $>$ environmental risk. Because the development of the new energy vehicle industry is at an early stage in Jiangsu province, various stakeholders, especially consumers, cannot fully recognize and accept this type of automobile; therefore, the industry faces high social-cognitive and industry-transformation risks. In addition, owing to the limited mileage, the battery technology has not achieved a fundamental breakthrough, a small number of charging piles, so the industry is facing a greater risk of international competitiveness. Moreover, the discontinuity and timeliness of policy formulation and the inconsistency between various policies lead to higher industry-policy risk in Jiangsu. Although the environmental risk is the lowest among exogenous risks, it has the greatest impact. This is because the environment is of great significance to the development of the company. The power batteries used in new energy vehicles are now mainly lithium-ion batteries. If discarded batteries are not properly recycled, destroyed, and stored, there is greater environmental pollution. However, the current battery-recycling system is imperfect in Jiangsu. Moreover, the technology of battery recycling is insufficient. Therefore, although Jiangsu has become the national pilot area for the recycling of new energy vehicle power batteries in China, the risk still exists. The regional ecological environment is also an important factor for industrial development, but it is fragile in Jiangsu province. Simultaneously, the imperfect legal system and economic fluctuations in Jiangsu are also the important factors that lead to high industrial environmental risk.

4.5.3. Industry Comprehensive Risk Analysis. The risk expectation of the new energy automobile industry in Jiangsu was 2.8849 . The industry comprehensive risk cloud map is located between the medium- and higher-medium-risk cloud maps. Therefore, the industry's comprehensive risk level is higher than medium risk, which is close to a higher medium risk. Moreover, the impact of exogenous risk on the new energy vehicle industry risk is greater than that of endogenous risk, as shown in Table 4.

\subsection{Suggestions}

4.6.1. Improvement of the Industry's Own Ability to Resist Risk. As can be seen from Figure 13, the development of the new energy automobile industry in Jiangsu faces a series of high endogenous risks, such as technological-innovation, production-cost, and financing risks. Therefore, industry enterprises should improve their ability to resist risk [52]. First, accelerating technical innovation and strengthening the core competitiveness of enterprises are necessary. The core competitiveness of the new energy automobile industry lies in its innovative technology and excellent product quality. However, the development of battery technology in the new energy automobile industry of Jiangsu has run into a bottleneck, and the industry faces higher technical risk. Therefore, in order to provide a good environmental foundation for technological innovation, the industrial science and technology innovation system and platform should be perfected [53]. Furthermore, cooperation in technology research and development between international and domestic enterprises should be strengthened to improve the efficiency of industrial research and development. Second, we should take some measures to expand financing channels. The new energy automobile industry is capitalintensive. Compared with other industrial enterprises, it has the characteristics of high production costs, high unit value, and high fixed cost investment. Hence, the development of the industry must be supported by strong capital. It is necessary to improve the new energy vehicle financial-service system to meet the financing needs of new energy vehicles such as production, operation, and consumption. Vigorously expanding financing channels requires the government to have a certain policy tendency towards industrial funds, which means increasing investment in research and development, and innovation. Moreover, it is necessary to guide social capital to participate in new energy vehicle production and operation services and especially encourage nonbank financial institutions to increase their efforts to support the financing of the new energy vehicle industry [54]. Lastly, the construction of industrial standardization needs to be sped up. Industrial standardization, as a crucial technical support for the development of new energy automobile industry, plays an important role in risk aversion. The construction of industrial standardization in the new energy automobile industry needs to be coordinated and managed by special government agencies to establish sound industrial standards and enhance the maneuverability and practicability of these standards. The establishment of standards that restrict the further development of industry must be optimized as soon as possible, including battery size, charging voltage, communication protocol, and the design of charging stations, so as to provide the support in technical 
standards and regulations for large-scale demonstration and industrialization.

4.6.2. Reduction of the Policy Risk in New Energy Vehicle Industry. Although policy risk is not the highest, its impact on the entire industry risk is very important (Figure 13). It is necessary to reduce policy risk. The new energy automobile enterprises of Jiangsu are overly dependent on policy support. In order to enhance the core capabilities of the industry, the role of market mechanism must be prominent [55]. First, it is important to clarify market-oriented and policy-guided development ideas. New energy vehicle industry policy in Jiangsu is strongly dominated, and industrial enterprises are more dependent on policy. Policy discontinuity, delay, and instability have a great impact on industrial enterprises. Therefore, clarifying development ideas is extremely important to reduce new energy vehicle industry policy risk in Jiangsu. Second, it is necessary to fully implement market-oriented and policy-guided development ideas. Policy should start from the cultivation of a favorable development environment for new energy automobiles and gradually establish a dominant position in the market. Guidance policy is formulated to synthesize production, learning, scientific research, practices and applications, and other social forces to build an environment for the development of the new energy automobile industry with Chinese characteristics and thus promote the rapid and healthy development of a new domestic electric-vehicle industry. Lastly, an evaluation mechanism should be established to evaluate policy-implementation effects. The implementation of policy should be evaluated regularly, and effectiveness evaluation and adjustment research should be carried out after the implementation of policy so as to continually optimize relevant measures.

4.6.3. Building Industry's Soft Environment. According to weight calculation, although environmental risk is higher than medium risk, impact on overall industry risk is the greatest. Hence, environmental risk must be reduced in order to reduce industrial risk. First, we build the industrial cultural environment. In order to make consumers feel the effect of low-carbon transportation on the environment, it is necessary to guide the whole society, and especially consumers, to establish values and consumption views that are compatible with low-carbon consumption. We should encourage low-carbon consumption and guide automotive consumers to pursue green and healthy orientation. The media, public opinion, and other ways to improve awareness and acceptance of new energy vehicles in the whole society should be utilized. Second, the legal environment should be improved, on one hand, incorporating new energy vehicle industry policies into the content of relevant laws to indirectly guide and control corporate behavior. On the other hand, legal means should be used to ensure that new energy vehicle policies can be effectively implemented. Third, power-battery storage and recycling environments need to be improved. First, it is necessary to promote automobile manufacturers to accelerate the establishment of recycling channels for used power batteries and then ensure that the responsibility extension system of producers is fully implemented. Second, traceability management could be used to accelerate the integration of the industrial chain. In order to carry out full life-cycle traceability management, we should have a unified code of power-storage batteries. Third, we should cultivate benchmarking enterprises for powerbattery recycling and explore the formation of a diversified recycling model with strong technical economy and resource-friendly environment. Lastly, ethical cultural-restraint and risk-management mechanisms to avoid ecological risk should be established in order to reduce regional ecological risk.

4.6.4. Improvement of the Risk-Sharing Mechanism. On the basis of the above analysis, the market risk of the new energy automobile industry in Jiangsu is at the higher-medium-risk level, so it is necessary to establish and improve the risktaking mechanism to guarantee the mutual benefit and win-win result of stakeholders involved in the new energy automobile industry to reduce market risk. First, establish and optimize a reasonable risk-taking mechanism. Building a reasonable benefit- and risk-sharing mechanism is necessary, since different stakeholders involved in new energy automobile industry have different risks. Second, the business model of new energy automobile industry should be innovated. From the perspective of the whole industry chain, the value chain should be reorganized to understand the course of value creation and the situation of input and output in all links and subjects. Then, a reasonable interest distribution mechanism and an open and sustainable operation mechanism for infrastructures should be formed. Additionally, increasing the level of consumer recognition is also the measure to reduce market risk. On the basis of the above research analysis, the risk of consumer cognition is higher than medium. Most buyers of new energy automobiles have concerns about the comfort, safety, charging procedures, and supporting the infrastructure of new energy electric automobiles. Hence, it is necessary to strengthen publicity efforts, carry out more offline experiencing programs, and guide consumers to experience and accept new energy automobiles.

\section{Conclusion}

With a large amount of greenhouse-gas emissions, global warming, and the deterioration of the global ecological environment, it is necessary to develop the new energy vehicle industry. However, the new energy vehicle industry is facing high risk. There is currently no comprehensive assessment method to quantitatively evaluate industry risk. Therefore, an evaluation model that can effectively evaluate the industrial risk situation was established in this article. The cloud model was applied to the risk assessment of the new energy automobile industry, and characteristic parameters were used to reflect the size of the industry risk. Compared with traditional risk-assessment methods, such as probabilistic risk assessment, score, risk matrix, Petri Nets, 
TOPSIS, fuzzy comprehensive evaluation, and rough-set theory, the cloud model can better reflect the ambiguity and randomness of each risk factor, guaranteeing the objectivity and accuracy of the assessment results.

This article first analyzed the industry risk-formation mechanism from endogenous and exogenous perspectives. Combining expert analysis with fuzzy statistical methods, the endogenous- and exogenous-risk-evaluation factors were screened out to construct an industry risk-evaluation system. Then, it applied the entropy weight-cloud model to evaluate the industry risk. The entropy weight method was used to determine the weight of each risk-evaluation index system, and the one-dimensional normal cloud model was used to evaluate industry risk. The forward cloud generator was used to repeatedly calculate the result of the cloud model, and the cloud drop distribution map of each level of risk factor was depicted. The risk level was upgraded from qualitative to quantitative, which provided a more accurate and intuitive basis for industry risk assessment. In the calculation process, this paper drew a cloud map of each risk impact factor and put it into the risk level cloud map for evaluation and analysis, more intuitively and visually analyzing the risk level to make results more convincing. Lastly, the risk of the new energy vehicle industry in Jiangsu in July 2019 was evaluated. On the basis of the empirical-analysis results, the industry-risk level of the new energy automobile industry was higher than medium and close to a higher medium. The impact of exogenous risk on the new energy vehicle industry was greater than that of endogenous risk. A series of suggestions, such as improving the industry's own ability, building the industry's own soft environment, reducing policy risk, and the improvement of the risk-sharing mechanism, were given for preventing industry risk.

This paper used the one-dimensional normal cloud model to evaluate industry risk, which can better reflect the ambiguity and randomness of each risk factor, guaranteeing the objectivity and accuracy of the assessment results. However, the rule generator of the one-dimensional normal cloud model can only represent the situation of single-input and single-output data. Industry risk has multidimensional attributes, and there is a coupling relationship between multidimensional attributes. Therefore, the multidimensional cloud model urgently needs to be discussed and researched.

\section{Data Availability}

The data used to support the findings of this study are available from the corresponding author upon request.

\section{Conflicts of Interest}

The authors declare no conflicts of interest.

\section{Authors' Contributions}

Conceptualization was done by Y. C. and Y. B.; formal analysis was carried out by R. W.; investigation was conducted by L. W.; methodology was done by Y. C. and L. W.; writing of original draft was performed by Y. C.; review and editing was carried out by R. W.

\section{Acknowledgments}

This research was funded by the Social Science Research in Colleges and Universities of Jiangsu Province, Research on Risk Assessment of Jiangsu New Energy Automobile Industry based on Entropy Weight-Cloud Model (2018SJA2084).

\section{References}

[1] J. Xie, J. Li, F. Yang, and L. Liang, "Decision-making and coordination optimized for multi-stage closed-loop supply chain of new energy vehicle," Journal of Industrial Engineering/Engineering Management, vol. 34, no. 5, pp. 180-193, 2020.

[2] Y. Cao, "Research on the development of new energy automobile industry in Jiangsu province," Value Engineering, vol. 38, no. 22, pp. 75-77, 2019.

[3] Y.-T. Li, M.-H. Huang, and D.-Z. Chen, "Positioning and shifting of technology focus for integrated device manufacturers by patent perspectives," Technological Forecasting and Social Change, vol. 81, pp. 363-375, 2014.

[4] M. Christopn, "A delphi-based risk analysis-identifying and assessing future challenges for supply chain security in a mufti-stakeholder environment," Technological Forecasting and Social Change, vol. 80, pp. 1815-1833, 2013.

[5] H. Liu and Y. Cheng, "Research on the key demand risk factors of China's new energy vehicles," Science and Technology Management Research, vol. 34, no. 19, pp. 217-223, 2014.

[6] C. Wang, J. Sun, L. Zuo, and H. Song, "Evaluation of global supply risk of critical minerals for new energy vehicles," China Science and Technology Forum, vol. 4, pp. 83-93, 2018.

[7] S. Zhang and W. Sun, "Evaluation of new energy vehicle venture capital project based on G1-entropy-TOPSIS," World Sci-Tech ReD, vol. 40, no. 2, p. 172, 2018.

[8] W. Chen, W. Zhou, and Y. Lang, "Research on clustering structure, betweenness and anti-risk capacity of innovation network of cluster-a case on industrial cluster of new energy vehicles in the northeast China," Management Review, vol. 27, no. 10, pp. 204-217, 2015.

[9] J. Wu and J. Liu, "Risks and coping tactics during evolution of new energy vehicle innovation ecosystem: case of BYD new energy vehicles," Science \& Technology Progress and Policy, vol. 33, no. 3, pp. 72-77, 2016.

[10] X. Su, Y. Zhuang, and H. Dai, "Study on risk of new energy vehicle burning-explosion and control measure," China Safety Science Journal, vol. 28, no. 5, pp. 92-98, 2018.

[11] F. Wang, J. Liao, D. Mao et al., "Aquatic quality criteria and ecological risk assessment for lead in typical waters of China," Asian Journal of Ecotoxicology, vol. 12, no. 3, pp. 434-445, 2017.

[12] D. Hubbard and D. Evans, "Problems with scoring methods and ordinal scales in risk assessment," IBM Journal of Research and Development, vol. 54, no. 3, 2010.

[13] J. Xiong, Q. Lu, Z. Li, G. Jiang, and Z. Xiong, "Logistic model of risk assessment based on big sampling data of elevator," Journal of Applied Statistics and Management, vol. 36, no. 4, pp. 599-608, 2017. 
[14] M. Caterino, M. Fera, R. Macchiaroli, and A. Lambiase, "Appraisal of a new safety assessment method using the petri nets for the machines safety," IFAC-PapersOnLine, vol. 51, no. 11, pp. 933-938, 2018.

[15] N. Xu and L. Feng, "Fuzzy comprehensive evaluation of network financing risk of small and micro-technological enterprises based on analytic hierarchy process," Science and Technology Management Research, vol. 39, no. 20, pp. 30-38, 2019.

[16] S. Zhu and Y. Qiu, "Risk assessment for suppliers in the air subcontracting production based on the entropy weight and TOPSIS," Systems Engineering, vol. 38, no. 1, pp. 154-158, 2020.

[17] W. Li, Risk Assessment of Power Systems: Models, Methods, and Applications, John Wiley \& Sons, Hoboken, NJ, USA, 2014.

[18] J. Wu, "E-commerce credit risk assessment based on rough set and support vector regression," Statistics and Decision, vol. 35, no. 1, pp. 51-54, 2019.

[19] R. Dahlgren, C. Chen-Ching Liu, and J. Lawarree, "Risk assessment in energy trading," IEEE Transactions on Power Systems, vol. 18, no. 2, pp. 503-511, 2003.

[20] S. Zhang, C. Xu, and Y. An, "Study on the risk evaluation approach based on cloud model," Journal of University of Electronic Science and Technology of China, vol. 42, no. 1, pp. 92-97, 2013.

[21] W. H. Zhang and S. F. Chen, "Research on mixed index hierarchical fuzzy decision-making method," Journal of Management Science, vol. 1, pp. 10-14, 2005.

[22] X. Liu, Z. Liu, and G. Wu, "Regional power grid security risk assessment based on FAHP and cloud model," Journal of Kunming University of Science and Technology, vol. 43, no. 4, pp. 71-77, 2018.

[23] D. Hou, H. Jiang, and H. Zhou, "Evaluation of policy-oriented agricultural insurance implementation effect in Heilongjiang province based on entropy weight-normal cloud model," Journal of Arid Land Resources and Environment, vol. 34, no. 4, pp. 33-38, 2020.

[24] Z. Lu, X. Sun, Y. Wang, and C. Xu, "Green supplier selection in straw biomass industry based on cloud model and possibility degree," Journal of Cleaner Production, vol. 209, pp. 995-1005, 2019.

[25] X. Shen, H. Yang, and C. Duan, "An analytical method of data mining on voltage sag based on gray target theory and cloud model," Power System Technology, vol. 43, no. 2, pp. 722-731, 2019.

[26] S. He, P. Pan, H. Wang, and L. Yu, "Research on ecological environment evaluation of agricultural land based on PSR and cloud theory," Journal of Natural Resources, vol. 26, no. 8, pp. 1346-1352, 2011.

[27] W. Ding, J. Wang, Z. Guan, and Q. Shi, "Minimum attribute reduction enhancing algorithm based on quantum cloud model evolution," Journal of Southeast University (Natural Science Edition), vol. 43, no. 2, pp. 290-295, 2013.

[28] D. Yang, D. Cui, and G. Cai, "A maximum power point tracking technology for fuel cells using cloud model based intelligent controller," Transactions of China Electrotechnical Society, vol. 33, no. 14, pp. 3362-3370, 2018.

[29] R. Guo, G. Chen, X. Zhao, J. Huo, and D. Fan, "Evaluation of Chinese train control system operational safety based on cloud model and uncertain analytic hierarchy process," Journal of the China Railway Society, vol. 38, no. 11, pp. 69-74, 2016.

[30] H. Tian and Q. Sun, "Research on capability evaluation of green technology innovation of garmakers based on cloud model," Management Review, vol. 32, no. 2, pp. 102-114, 2020.
[31] L. Wang and Y. Wang, "Assessment on industrial chain risk of equipment manufacturing industry based on cloud model," Technology Economics, vol. 35, no. 2, pp. 80-87, 2016.

[32] W. Xiong, H. Zhang, Z. Xie, X. Han, Z. Li, and X. Lin, "Risk assessment of power transformer potential fault based on cloud theory and entropy weight method," Electric Power Automation Equipment, vol. 38, no. 8, pp. 125-130, 2018.

[33] W. Yang, S. Yang, and Q. Xie, "Study on slope risk assessment method based on cloud model and its application," Huazhong University of Science and Technology, vol. 46, no. 4, pp. 30-34, 2018.

[34] L. Liang, G. Xing, and F. Wu, "Durability risk assessment of stay cables based on cloud theory," Journal of Northeastern University, vol. 40, no. 3, pp. 425-429, 2019.

[35] L. Zhou, Z. Yuan, C. Xu, and X. Tang, "Research on cost risk management of water conservancy project based on modified cloud model," Water Resource and Power, vol. 37, no. 4, pp. 152-154, 2019.

[36] J. Dong, D. X. Wang, D. R. Liu, P. Ainiwaer, and L. P. Nie, "Operation health assessment of power market based on improved matter-element extension cloud model," Sustainability, vol. 11, no. 19, Article ID 11195470, 2019.

[37] X. Zou and L. Tian, "Optimal site selection of electric vehicle charging stations based on a cloud model and the PROMETHEE method," Energies, vol. 3, pp. 182-185, 2016.

[38] F. Liang, "Quality assessment of tax revenue based on AHP and entropy weight method-an empirical research base on the data of tax system in 2011," Taxation and Economy, vol. 5, pp. 70-78, 2013.

[39] Z. Chen and Z. Dai, "Improved cloud model for stability evaluation of reservoir slopes," Journal of Engineering Geology, vol. 28, no. 3, pp. 619-625, 2020.

[40] Y. Liu, Z. Xu, H. Fu, G. Li, and S. Gao, "Insulation condition assessment method of power transformer based on improved extension cloud theory with optimal cloud entropy," High Voltage Engineering February, vol. 46, no. 2, pp. 397-405, 2020.

[41] Y. Yao and N. Lin, "A research about venture capital involvement in China new energy industry," SciencesTechnology Progress and Policy, vol. 3, pp. 61-64, 2012.

[42] J. Chen, Y. Liu, and L. Wang, "Research on coupling coordination development for photovoltaic agriculture system in China," Sustainability, vol. 11, no. 4, p. 1065, 2019.

[43] L. Wang, Y. Wang, and J. Chen, "Assessment of the ecological niche of photovoltaic agriculture in China," Sustainability, vol. 11, no. 8, p. 2268, 2019.

[44] L. Wang, Y. Wang, and J. Chen, "Social self-organization evolution path of photovoltaic industry chain system," Revista de Cercetare si Interventie Sociala, vol. 65, pp. 338-353, 2019.

[45] R. Wang, H. Zameer, Y. Feng, Z. Jiao, L. Xu, and A. Gedikli, "Revisiting Chinese resource curse hypothesis based on spatial spillover effect: a fresh evidence," Resources Policy, vol. 64, Article ID 101521, 2019.

[46] H. Shen, Y. Hou, H. Fu, and N. Li, "Research on risk assessment method for government-leading regional strategic emerging industry: from the perspective of industry competitive intelligence," Journal of Intelligence, vol. 35, no. 6, pp. 109-114, 2016.

[47] M. Li and R. Bao, "The analysis of risk investment supports strategic emerging industry development analysis," Macroeconomic Research, vol. 5, pp. 123-128, 2016.

[48] L. Wang and Z. Jiang, "Study on financial risk assessment of p2p platforms based on structural entropy weight method," Journal of Systems Science, vol. 2, pp. 95-99, 2020. 
[49] C. She, Z. Zhang, P. Iiu, and F. Sun, "Overview of the application of big data analysis technology in new energy vehicle industry: based on operating big data of new energy vehicle," Chinese Journal Mechanical Engineering, vol. 55, no. 20, pp. 3-16, 2019.

[50] J. Zhang, X. Yin, C. Guan, and H. Zhang, "Financial status and technology innovation of strategic emerging industry: a case of new energy industry," Journal of Nanjing Tech University (Social Science Edition), vol. 18, no. 5, pp. 102-110, 2019.

[51] $\mathrm{F}$. $\mathrm{Hu}$, "Reaearch on Jiangsu new energy vehicle industry based on porter competitive model," Special Zone Economy, vol. 9, pp. 44-46, 2016.

[52] S. Pan, B. Li, and H. Nie, "Comprehensive evaluation and analysis of restricting factors of sustainable development of China's new energy automobile industry from the perspective of innovative ecosystem," Science and Technology Management Research, vol. 39, no. 22, pp. 41-47, 2019.

[53] W. He, "Policy protection and niches construction in the new energy vehicles industry-an analysis based on a multi-level industry evolution," Science Research Management, vol. 40, no. 9, pp. 139-148, 2019.

[54] K. Li, W. Lu, and C. Liang, "Review and prospect of China's new energy vehicle power battery from the perspective of management," Science and Technology Management Research, vol. 40, no. 5, pp. 173-177, 2020.

[55] S. Ding, "The logic and countermeasures of the generation of ecological riskin the process of industrialization," China Social Science University Humanity, vol. 1, pp. 72-77, 2020. 